This is the final peer-reviewed accepted manuscript of:

A. Mariani, S. Kandeepan and A. Giorgetti, "Periodic Spectrum Sensing With NonContinuous Primary User Transmissions" in IEEE Transactions on Wireless Communications, vol. 14, no. 3, pp. 1636-1649, March 2015

The final published version is available online at:

https://doi.org/10.1109/TWC.2014.2371024

Rights / License:

The terms and conditions for the reuse of this version of the manuscript are specified in the publishing policy. For all terms of use and more information see the publisher's website.

This item was downloaded from IRIS Università di Bologna (https://cris.unibo.it/)

When citing, please refer to the published version. 


\title{
Periodic Spectrum Sensing with Non-Continuous Primary User Transmissions
}

\author{
Andrea Mariani, Member, IEEE, Sithamparanathan Kandeepan, Senior Member, IEEE, and \\ Andrea Giorgetti, Senior Member, IEEE
}

\begin{abstract}
In this paper we present a thorough study of spectrum sensing performance in cognitive radio (CR) scenarios where the primary user (PU) transmission is not continuous. In particular, we consider a sensing scheme in which the spectrum is monitored periodically for a fraction of time. In such a situation, sensing is affected by common detection impairments, including noise and fading, as well as by the PU temporal behavior. It is thus necessary to properly design periodic sensing parameters to balance between sensing overhead and detection performance. In this context, we derive a comprehensive analytical framework which accounts for detector performance, presence of noise and fading, PU temporal statistics and periodic sensing. The analysis allows to express the detection and false alarm probabilities in closed-forms to capture an explicit relationship between the PU temporal statistic and periodic sensing parameters. Our results show that the temporal behavior of the PU have a significant impact on the detection performance, and therefore a proper design of the sensing parameters is important. Based on our analysis we propose useful strategies to design effective periodic sensing.
\end{abstract}

Index Terms-Cognitive radio, detection, periodic sensing, spectral occupancy, spectrum sensing.

\section{INTRODUCTION}

$\mathbf{T}$ HE SPECTRUM is a limited resource, and the ever growing demand for frequency bands has brought about the problems related to its shortage. Recent studies have emphasized that the real problem does not lie in the scarcity of resources available, but rather in the fact that they are poorly managed [1]. These considerations have lead to the development and evolution of the concept of cognitive radio (CR), further supported by the radio regulatory bodies around the world [1]-[3]. A CR device, by intelligently sensing and learning from the radio environment, uses spectrum opportunities as a secondary user (SU) whilst giving higher priority to the primary users (PUs) of the spectrum.

A. Mariani and A. Giorgetti are with CIRI-ICT/DEI, University of Bologna, via Venezia 52, 47521 Cesena (FC), Italy (e-mail: a.mariani@unibo.it, andrea.giorgetti@unibo.it).

S. Kandeepan is with RMIT University, Melbourne, and also with the National ICT Australia (NICTA), Melbourne (e-mail: kandeepan@ieee.org). NICTA is funded by the Australian Government as represented by the Department of Broadband, Communications and the Digital Economy and the Australian Research Council through the ICT Centre of Excellence program.

This work was presented, in part, at the IEEE Int. Conf. on Comm. (ICC), Cape Town, South Africa, May 2010, and at the IEEE Global Comm. Conf. (GLOBECOM), Honolulu, USA, Nov. 2009. The research work was partly funded by the European Unions Seventh Framework Programme (FP7/20072013) under grant agreement CONCERTO n. 288502 and under the grant agreement ABSOLUTE n. 318632, and in part by the Italian Ministry of Education, Universities and Research (MIUR) under Research Projects of Significant National Interest PRIN 2011 GRETA.
Spectrum sensing is one of the key functionalities for opportunistic spectrum access, extensively studied in the recent literature [3]-[12]. Many spectrum sensing algorithms have been proposed, starting from most simple approaches, such as the energy detector (ED), that estimates the received signal energy and compares it to a decision threshold [1], [4], [5]. The unique impairment of the ED is that it requires the estimation of noise power level (see [5] for further details). If some features of the PU signal to be detected are known, they can be used for detection purposes, implementing algorithms such as correlation-based detection or cyclostationary detectors [1]. Other algorithms exploit diversity strategies, such as multiple antennas or cooperation among different nodes. In this context, the most popular approaches are based on the observation of the eigenvalues of the sample covariance matrix [6], [7]. For a more complete overview on spectrum sensing algorithms refer to [8] and [3, Chapter 1].

Considering the spectral occupancy in the time domain, there are mainly two scenarios that may be considered. The first one is in presence of continuous PU transmissions, such as television broadcasting in TV white spaces, or non continuous transmissions with high occupancy rates [32]. In these contexts the SU typically senses the spectrum in order to discover spectrum opportunities in the space and frequency domains. A different situation occurs in presence of non-continuous PU transmissions with a low occupancy rate such as wireless microphones, voice-based services in cellular systems, or packetbased wireless communications. In these cases, the variability of spectrum occupancy in time motivates the search for temporal spectrum holes [13]-[15]. To cope with non-continuous transmissions, periodic spectrum sensing has been proposed and investigated in specific contexts. Spectrum occupancy estimation in time-domain has been first analyzed in [16] using an asymptotical approach. The sensing requirements and the detection performance with periodic frequency scanning have been derived in [17], and in [18] the authors study scheduling for periodic sensing and the differences between the energy and feature based techniques. In [19] periodic sensing has been proposed in the practical context of the IEEE 802.22 standard with $1 \mathrm{~ms}$ sensing duration every $40 \mathrm{~ms}$. In [15] the problem of temporal spectrum holes discovery has been studied addressing the problem of learning the PU channel usage pattern, but assuming a sensing process with zero error probability. However, a comprehensive and systematic analysis of periodic sensing has not been addressed thoroughly.

In this paper, we formulate a general framework for temporal spectrum sensing, including PU non-continuous transmis- 


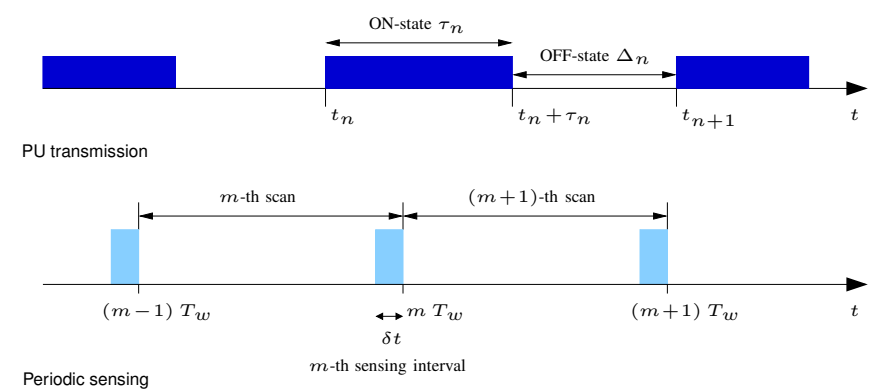

Fig. 1. Primary user temporal behavior and periodic spectrum sensing by the $\mathrm{CR}$.

sions, periodic sensing, noise, and fading. In particular, the contributions of the paper are as follows.

- We provide general expressions of the temporal detection and false alarm probabilities that capture the explicit relationship between detector performance, noise effect, fading impact, PU temporal statistic and periodic sensing parameters.

- The analysis extends previously proposed studies, providing a formulation that can be applied to different detection algorithms and different PU temporal statistics.

- To investigate different scenarios, we derive the performance of three sensing tasks corresponding to the detection of a specific PU transmission, the detection of the PU using a single observation, and using multiple scans.

- The case in which sensing is based on ED and the PU is characterized by exponential inter-arrival and hold-time distributions is discussed in detail providing closed-form expressions for the probabilities of temporal detection and false alarm.

Our results show that the temporal statistic of the PU has a significant impact on the detection performance, and therefore a proper design of periodic sensing parameters is crucial. Finally, based on our analysis, we propose useful strategies to design effective periodic sensing in the CR context.

The rest of the paper is organized as follows. In Section II we present the temporal sensing framework providing general forms of the detection metrics. In Section III we analyze the case in which ED is adopted in presence of a PU with exponential distributed arrival time. In Sections IV and V we analyze periodic sensing based on the temporal statistic of the PU for constant and random hold-time cases, while in Section VI we present the design strategies, based on the periodic sensing framework proposed, and some numerical results. Finally, in Section VII we draw some concluding remarks.

\section{TEMPoral SENSING FRAMEWORK}

In this section, we present the system model considered for the analysis of periodic sensing in the presence of noncontinuous PU transmissions, and the definition of the detection metrics adopted. The PU temporal behavior is statistically modeled as a stationary ON-OFF random process with two states, where the $\mathrm{ON}$ state represents the PU transmission and the OFF state represents the PU inactivity. The $\mathrm{ON}$ and $\mathrm{OFF}$ period durations are denoted with $\tau_{n}$ and $\Delta_{n}$, respectively, with $n \in \mathbb{N}$. Fig. 1 depicts an example of temporal scenario with non-continuous PU transmission, where the SU asynchronously observes the channel within sensing intervals of duration $\delta t$. We refer to the period between the ending of two consecutive sensing intervals, with duration $T_{w}$, as the sensing scan.

\section{A. Periodic Spectrum Sensing}

The temporal spectrum sensing performance can be studied starting from the definition of three temporal sensing tasks (TSTs):

- TST- $n m$ : detect the $n$th PU transmission within the $m$ th sensing scan;

- TST-S: detect any PU transmission within a single scan;

- TST-M: detect any PU transmission using multiple scans.

The first TST can be adopted for the design of the periodic sensing parameters, and for quantifying the impact of periodic sensing in terms of latency of the detection process, as described in Section VI. Moreover, the TST- $n m$ represents an elementary task useful for the study of the latter two. The TST-S aims at quantifying the overall sensing capability to detect a PU within a single sensing scan. On the basis of this single observation the SU decides which behavior to adopt, such as, e.g., whether to transmit, and how to plan future actions, such as sensing intervals scheduling. ${ }^{1}$ Another relevant scenario is represented by the situation in which the $\mathrm{SU}$ is interested in knowing if a PU is present to avoid using the channel for a relatively long period (band dropping) [1]. The SU performs sensing in different channels allocating different sensing periods within the same scan in time-division, but instead of making a decision at the end of each sensing interval, consecutive $M$ scans can be monitored and a unique decision is made at the end. This situation is represented by TST-M, where an higher detection probability is expected and specific design rules are presented in the next sections. Note that TST-S and TST-M can be also combined in such a way that a first decision on whether to use a channel or not can be performed with TST-M and then, on a scan-by-scan basis, the selected channel can be monitored with TST-S. We remark that TST-S and TST-M directly provide a decision on the occupancy state of the channel, while TST- $n m$ is useful for the analysis of periodic sensing and to derive a design strategy that accounts for detection latency.

Considering the presence of non-continuous PU transmissions, the sensing process is affected by the fact that PU transmissions are not always overlapped with the sensing intervals. Thus, for studying this temporal sensing problem, it is useful to define two events, $\mathcal{S}$ and $\mathcal{T}$, associated to a PU transmission: ${ }^{2}$

\footnotetext{
${ }^{1}$ Within the paper, we do not make assumptions on the SU behavior outside the sensing intervals. The time between two sensing intervals could be used, e.g., for transmitting SU data when the channel has been declared free, or, alternatively, to sense other channels.

${ }^{2}$ In the following, we use the notations $\overline{\mathcal{S}}$ and $\overline{\mathcal{T}}$ to denote the corresponding complementary events.
} 


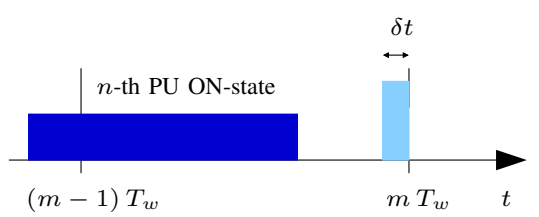

(a) $\mathcal{T}_{n, m}$ and $\overline{\mathcal{S}}_{n, m}$

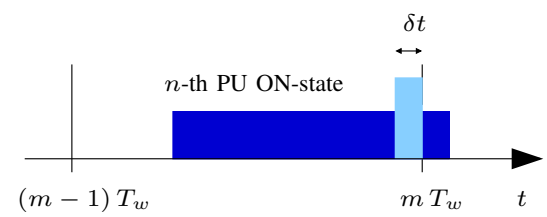

(b) $\mathcal{T}_{n, m}$ and $\mathcal{S}_{n, m}$

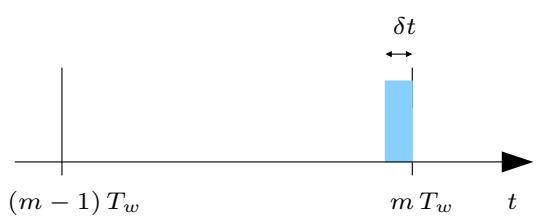

(c) $\overline{\mathcal{T}}_{n, m}$

Fig. 2. Temporal events related to the temporal sensing tasks.

- $\mathcal{S}$ : the PU transmission is overlapped with a sensing interval;

- $\mathcal{T}$ : the PU transmission is in a scan period.

In particular, with reference to the TSTs defined above, we adopt the notation:

- $\mathcal{S}_{n, m}$ and $\mathcal{T}_{n, m}$, for the TST- $n m$, to indicate the presence of the $n$th PU transmission within the $m$ th sensing interval, and the presence of the $n$th PU transmission within the $m$ th sensing scan, respectively;

- $\mathcal{S}_{m}$ and $\mathcal{T}_{m}$, for the TST-S, to indicate the presence of at least one PU transmission within the $m$ th sensing interval, and the presence of at least one PU transmission within the $m$ th sensing scan, respectively;

- $\mathcal{S}_{M}$ and $\mathcal{T}_{M}$, for the TST-M, to indicate the presence of at least one PU transmission within $M$ sensing intervals, and the presence of at least one PU transmission within $M$ sensing scans, respectively.

Throughout the paper we use the notation $\mathcal{S}$ and $\mathcal{T}$ to indicate either $\mathcal{S}_{n, m}$ and $\mathcal{T}_{n, m}$, or $\mathcal{S}_{m}$ and $\mathcal{T}_{m}$, or $\mathcal{S}_{M}$ and $\mathcal{T}_{M}$, to maintain a general approach and avoid repetitions, and we specialize the analysis using the specific symbols, when necessary. A graphical representation of the possible events for TST- $n m$ is provided in Fig. 2.

In analyzing the periodic sensing it is useful to consider two situations: a transient phase and a steady-state regime. The transient phase occurs when the SU starts monitoring the channel and consists in a situation in which the detection performance strongly depends on the sensing interval index $m$, while in the steady-state regime the detection performance is constant in time.

\section{B. Detection Metrics}

In this section, we discuss temporal sensing in a general framework, defining proper performance metrics. In the following sections, we explicitly calculate the detection performance in a specific case.

Signal detection is affected by noise, channel impairments and also by the non-continuous transmission of the PU. It is thus useful to separate their effects to better understand their impact on detection performance and to derive a general framework which accounts for PU temporal behavior and periodic sensing parameters. In light of this approach, we define two metrics namely, temporal sensing probability and temporal detection probability.

Let us define the temporal sensing probability as $P_{\mathrm{TS}}=$ $\mathbb{P}\{\mathcal{S} \mid \mathcal{T}\}$, which can be rewritten, using Bayes' theorem, as
$P_{\mathrm{TS}}=\mathbb{P}\{\mathcal{S} \cap \mathcal{T}\} / \mathbb{P}\{\mathcal{T}\}$. Given that $\mathcal{S} \subset \mathcal{T}$, such probability can be expressed as [20]

$$
P_{\mathrm{TS}}=\frac{\mathbb{P}\{\mathcal{S}\}}{\mathbb{P}\{\mathcal{T}\}} .
$$

As can be seen, $P_{\mathrm{TS}}$ only accounts for periodic sensing and non-continuous transmission of the PU, not including the detection process in the presence of noise and channel impairments. ${ }^{3}$

In the presence of noise, considering a general detector characterized by a decision statistic $\Lambda$ and a threshold $\xi$, the detection of a PU may occur in two cases: either because there is an overlap between the PU transmission and the sensing period with a correct detection (i.e., the event $\Lambda>\xi \mid \mathcal{S}$ ) or because there is a false alarm with no overlap between the PU transmission and the sensing period (i.e., the event $\Lambda>\xi \mid \overline{\mathcal{S}}$ ). Accordingly, we define the temporal detection probability as

$$
\begin{aligned}
P_{\mathrm{TD}}= & \mathbb{P}\{\Lambda>\xi \mid \mathcal{T}\}= \\
& \mathbb{P}\{\Lambda>\xi \mid \mathcal{S}\} \mathbb{P}\{\mathcal{S} \mid \mathcal{T}\}+\mathbb{P}\{\Lambda>\xi \mid \overline{\mathcal{S}}\} \mathbb{P}\{\overline{\mathcal{S}} \mid \mathcal{T}\} \\
= & P_{\mathrm{D}} \mathbb{P}\{\mathcal{S}\} / \mathbb{P}\{\mathcal{T}\}+P_{\mathrm{FA}}(1-\mathbb{P}\{\mathcal{S}\} / \mathbb{P}\{\mathcal{T}\}) \\
= & P_{\mathrm{D}} P_{\mathrm{TS}}+P_{\mathrm{FA}}\left(1-P_{\mathrm{TS}}\right)
\end{aligned}
$$

where $\mathbb{P}\{\Lambda>\xi \mid \mathcal{S}\}$ and $\mathbb{P}\{\Lambda>\xi \mid \overline{\mathcal{S}}\}$ are, respectively, the conventional probability of detection and probability of false alarm of the specific detector considered. ${ }^{4}$ The above definition of temporal detection incorporates both periodic sensing and detection, and is by no means in conflict to the usual definition of detection probability, where the event $\overline{\mathcal{T}}$ corresponds to the null hypothesis and $\mathcal{T}$ to the alternative hypothesis [29]. Note that this is the main difference between simple detection and temporal detection; the former wants to infer the occupancy state in the observed interval given that there is a transmission in that interval, while the latter wants to infer the occupancy state accounting for the non-continuous nature of PU transmissions within a sensing scan.

If the PU transmission is continuous, then $P_{\mathrm{TS}}=1$ and $P_{\mathrm{TD}}$ reduces to the detection probability, i.e., $P_{\mathrm{TD}}=P_{\mathrm{D}} .{ }^{5}$ On the other hand, when the detection probability is $P_{\mathrm{D}}=1$ and the false alarm probability is $P_{\mathrm{FA}}=0$ (a condition that can be approached for high signal-to-noise ratios (SNRs)) $P_{\mathrm{TD}}$ in (2) reduces to $P_{\text {TS }}$. Hence, temporal sensing probability can be interpreted as an asymptotic performance in the high

\footnotetext{
${ }^{3}$ Such metric can be interpreted as the overlap probability among the sensing periods and the PU transmissions.

${ }^{4}$ Also note that the events $\mathcal{S}$ and $\overline{\mathcal{S}}$ are often denoted as $\mathcal{H}_{1}$ and $\mathcal{H}_{0}$, respectively.

${ }^{5}$ This fact implies that the sensing scan duration, $T_{w}$, does no longer play any role, and periodic sensing looses its significance.
} 
SNR regime. This fact is intuitive, since when noise can be neglected, the performance depends only on the PU temporal behavior and periodic sensing parameters. Also note that in (2) noise and temporal overlap are clearly separated. This aspect is useful to understand temporal sensing dependence on PU temporal behavior and to design periodic sensing parameters, as discussed in the next sections.

Since $\overline{\mathcal{T}} \subset \overline{\mathcal{S}}$, the probability of false alarm in the presence of non-continuous PU transmission can be written as

$$
P_{\mathrm{FA}}=\mathbb{P}\{\Lambda>\xi \mid \overline{\mathcal{T}}\}=\mathbb{P}\{\Lambda>\xi \mid \overline{\mathcal{S}}\}
$$

which corresponds to the usual definition.

The detection probability over $M$ scans depends on how we combine the information collected from the $M$ sensing intervals. There are several data fusion strategies that can be used, generally classified as hard fusion schemes, that combine the results of hypothesis tests applied to each sensing interval, and soft fusion techniques, that combine the observations of different sensing intervals and use a global detection test.

Considering hard combining strategies, we adopt the OR rule, in which the CR decides for the presence of the PU if it is detected in any of the $M$ sensing intervals. ${ }^{6}$ The probability of detecting at least one transmission, given that at least one has occurred in the $M$ scans, can be written as

$$
P_{\mathrm{TD}}^{\mathrm{FU}}=\mathbb{P}\left\{\left(\bigcup_{m \in \Omega_{M}}\left\{\Lambda_{m}>\xi\right\}\right) \neq \emptyset \mid \mathcal{T}_{M}\right\}
$$

where $\Lambda_{m}$ is the test statistic in the $m$ th sensing interval and $\Omega_{M}$ is set of the indexes of $M$ consecutive sensing periods. Such expression can be simplified assuming the independence among different sensing periods, which is verified in particular when $\tau$ is small with respect to $T_{w} \cdot{ }^{7}$ Due to the independence assumption, $P_{\mathrm{TD}}^{\mathrm{FU}}$ can be expressed as

$$
P_{\mathrm{TD}}^{\mathrm{FU}}=1-\prod_{m \in \Omega_{M}}\left(1-\mathbb{P}\left\{\Lambda_{m}>\xi \mid \mathcal{T}_{M}\right\}\right)
$$

where the inner term $\mathbb{P}\left\{\Lambda_{m}>\xi \mid \mathcal{T}_{M}\right\}$ can be expanded similarly to (2) as

$$
\mathbb{P}\left\{\Lambda_{m}>\xi \mid \mathcal{T}_{M}\right\}=P_{\mathrm{D}} \mathbb{P}\left\{\mathcal{S}_{m} \mid \mathcal{T}_{M}\right\}+P_{\mathrm{FA}}\left(1-\mathbb{P}\left\{\mathcal{S}_{m} \mid \mathcal{T}_{M}\right\}\right) .
$$

Now, let us denote with $P_{\mathrm{S}}(m)=\mathbb{P}\left\{\mathcal{S}_{m}\right\}$ the probability of an overlap between a PU transmission and the $m$ th sensing interval, and with $P_{\mathrm{T}}^{\mathrm{M}}=\mathbb{P}\left\{\mathcal{T}_{M}\right\}$ the probability of at least one transmission within the $M$ scans. Considering that $\mathcal{S}_{m} \subset \mathcal{T}_{M}$, $\mathbb{P}\left\{\mathcal{S}_{m} \mid \mathcal{T}_{M}\right\}=\mathbb{P}\left\{\mathcal{S}_{m}\right\} / \mathbb{P}\left\{\mathcal{T}_{M}\right\}$ and the temporal detection probability (5) can be finally written in closed-form as

$$
P_{\mathrm{TD}}^{\mathrm{FU}}=1-\prod_{m \in \Omega_{M}}\left(1-P_{\mathrm{D}} \frac{P_{\mathrm{S}}(m)}{P_{\mathrm{T}}^{\mathrm{M}}}-P_{\mathrm{FA}}\left(1-\frac{P_{\mathrm{S}}(m)}{P_{\mathrm{T}}^{\mathrm{M}}}\right)\right) \text {. }
$$

\footnotetext{
${ }^{6} \mathrm{We}$ choose the OR rule because it is the most conservative from the PU protection perspective [1].

${ }^{7}$ As shown in Section VI, such approximation gives very good results compared with simulations.
}

Considering the high SNR regime, the temporal sensing probability can be derived from (7) as

$$
P_{\mathrm{TS}}^{\mathrm{FU}}=\mathbb{P}\left\{\mathcal{S}_{M} \mid \mathcal{T}_{M}\right\}=1-\prod_{m \in \Omega_{M}}\left(1-\frac{P_{\mathrm{S}}(m)}{P_{\mathrm{T}}^{\mathrm{M}}}\right) .
$$

According to (3), the false alarm probability is not affected by the PU temporal behavior, hence considering the OR-based fusion rule we have

$$
P_{\mathrm{FA}}^{\mathrm{FU}}=1-\left(1-P_{\mathrm{FA}}\right)^{M} .
$$

Considering the soft fusion schemes, the hypothesis test $\Lambda_{\mathrm{SF}}$ for the TST-M depends on the number of overlaps between the PU transmissions and the sensing intervals, denoted in the following as $K$. Thus the temporal detection probability for $\Lambda_{\mathrm{SF}}$ can be expressed as

$$
P_{\mathrm{TD}}^{\mathrm{FU}}=\mathbb{P}\left\{\Lambda_{\mathrm{SF}}>\xi \mid \mathcal{T}\right\}=\sum_{k=0}^{M} p_{k} \mathbb{P}\left\{\Lambda_{\mathrm{SF}}>\xi \mid K=k\right\}
$$

where $\xi$ is the decision threshold and $p_{k}=\mathbb{P}\{K=k\}$, as explained in Section III-B, is related to the temporal sensing probability. In fact, in the high SNR regime, $\Lambda_{\mathrm{SF}}$ is above the threshold when $K>0$, and thus from (10) we obtain the corresponding temporal sensing probability $P_{\mathrm{TS}}^{\mathrm{FU}}=\sum_{k=1}^{M} p_{k}$. The false alarm probability for the soft fusion schemes is given by $P_{\mathrm{FA}}^{\mathrm{FU}}=\mathbb{P}\left\{\Lambda_{\mathrm{SF}}>\xi \mid \overline{\mathcal{T}}\right\}$. In the next section, we discuss in detail the case where soft fusion is performed by equal gain combining (EGC) with energy detection.

\section{Temporal Detection Probability with Noise, FADING AND NON-CONTINUOUS PU TRANSMISSION}

The temporal sensing framework described in the previous section can be used in different practical case studies, by defining the PU ON-OFF statistics, the detector adopted and the fading model. Note that these aspects have different effects on the temporal detection metric. The PU temporal behavior, indeed, affects the temporal sensing probabilities $\left(P_{\mathrm{TS}}, \mathbb{P}\{\mathcal{S}\}\right.$, and $\mathbb{P}\{\mathcal{T}\}$ ), while the choice of the detector used and the fading model impacts $P_{\mathrm{D}}$ and $P_{\mathrm{FA}}$. In this section, we consider the case study in which ED is adopted in presence of a PU with exponential arrival time.

Let us model the OFF-periods duration $\Delta_{n}$ as exponentially distributed random variables (r.v.s) with mean $\mathbb{E}\left\{\Delta_{n}\right\}=$ $\bar{\Delta}=1 / \lambda$, where $\lambda$ is the vacancy rate. For the ON-periods we analyze two situations: 1) the constant hold-time case where the occupancy times $\tau_{n}$ are deterministic and equal, i.e., $\tau_{n}=\tau$; and 2) the random hold-time case where the occupancy times $\tau_{n}$ are exponentially distributed r.v.s with mean $\mathbb{E}\left\{\tau_{n}\right\}=\bar{\tau}=1 / \mu$, where $\mu$ is the occupancy rate. Such statistical description has been widely adopted to model spectrum holes such as in [15], [20]-[27], also confirmed by spectrum occupancy measurements in [27] and by monitored HSDPA traffic in [28].

Although the temporal sensing analysis can consider, in principle, any type of detection technique, we focus our attention on the ED-based spectrum sensing. This choice is motivated by the fact that ED is the reference detector adopted in CR literature due to its simplicity and the fact that it does 
not require a priori knowledge of the PU signal characteristics [1], [4], [5].

After bandpass filtering over a bandwidth $W$, the received signal $r(t)$, observed for $\delta t$ seconds, is down converted and sampled at frequency $W$, obtaining $N_{\mathrm{s}}=\delta t W$ complex samples $\left\{y_{j}\right\}_{j=0}^{N_{\mathrm{s}}-1} .8$ Hence, in the following we analyze the discrete time detection problem

$$
\begin{array}{ll}
\overline{\mathcal{S}}: & y_{j}=n_{j} \\
\mathcal{S}: & y_{j}=x_{j}+n_{j}
\end{array}
$$

with $j=0, \ldots, N_{\mathrm{s}}-1$, where $\left\{x_{j}\right\}_{j=0}^{N_{\mathrm{s}}-1}$ are the signal samples and $\left\{n_{j}\right\}_{j=0}^{N_{\mathrm{s}}-1}$ is a set of independent, identically distributed (i.i.d.) circularly symmetric complex Gaussian r.v.s with zero mean and variance $2 \sigma^{2}$, representing the additive white Gaussian noise (AWGN). The test statistic for the ED is given by [4]

$$
\Lambda=\frac{1}{2 \sigma^{2} N_{\mathrm{s}}} \sum_{j=0}^{N_{\mathrm{s}}-1}\left|y_{j}\right|^{2} \underset{\mathcal{\mathcal { S }}}{\stackrel{\mathcal{S}}{\gtrless}} \xi
$$

where the SNR in the hypothesis $\mathcal{S}$ is defined as $\rho=$ $1 /\left(2 \sigma^{2} N_{\mathrm{s}}\right) \sum_{j=0}^{N_{\mathrm{s}}-1}\left|x_{j}\right|^{2}$, and $\xi$ is the decision threshold.

Considering the AWGN scenario, it is well known that the test statistic (12) follows a chi squared distribution with $2 N_{\mathrm{s}}$ degrees of freedom and thus we have $[4]^{9}$

$$
\begin{aligned}
P_{\mathrm{D}}(\rho) & =\mathbb{P}\{\Lambda>\xi \mid \mathcal{S}\}=\mathcal{Q}_{N_{\mathrm{s}}}\left(\sqrt{2 N_{\mathrm{s}} \rho}, \sqrt{2 N_{\mathrm{s}} \xi}\right) \\
P_{\mathrm{FA}} & =\mathbb{P}\{\Lambda>\xi \mid \overline{\mathcal{S}}\}=\widetilde{\Gamma}\left(N_{\mathrm{s}}, N_{\mathrm{s}} \xi\right)
\end{aligned}
$$

where $\mathcal{Q}_{\alpha}(a, b) \quad=\quad \int_{b}^{\infty} u^{\alpha} \exp \left(-\left(u^{2}+\right.\right.$ $\left.\left.a^{2}\right) / 2\right) \mathcal{I}_{\alpha-1}(a u) / a^{\alpha-1} d u$ is the generalised Marcum $\mathrm{Q}$ function with $\mathcal{I}_{\alpha-1}(\cdot)$ being the modified Bessel function of first kind with order $\alpha-1$, and $\widetilde{\Gamma}(a, z) \triangleq \frac{1}{\Gamma(a)} \int_{z}^{\infty} x^{a-1} \exp (-x) d x$ is the regularized upper incomplete Gamma function with $\Gamma(\cdot)$ the Gamma function. When the PU signal is subject to slow fading, the SNR can be modelled by its probability density function (p.d.f.) $f_{\rho}(\cdot \mid \bar{\rho})$ with mean value $\bar{\rho}=\mathbb{E}\{\rho\}$. Therefore, the average detection probability can be computed as ${ }^{10}$

$$
\widetilde{P}_{\mathrm{D}}(\bar{\rho})=\int_{0}^{\infty} P_{\mathrm{D}}(h) f_{\rho}(h \mid \bar{\rho}) d h .
$$

In Section VI we consider the Rayleigh fading case where the corresponding detection probability can be found in closedform in [36, eq. (9)]. Similar expressions for Rice and Nakagami fading can be found in [36].

\footnotetext{
${ }^{8}$ Without loss of generality we consider $\delta t W$ integer [4].

${ }^{9}$ Because of the PU temporal behavior, the hypothesis $\mathcal{S}$ does not necessarily imply that the PU transmission is completely overlapped with the sensing interval. Thus, in general, the energy captured by the ED is a r.v. that depends on the amount of temporal overlap [32], [33]. However, since our interest is in finding temporal spectrum holes with good temporal resolution, it is reasonable to consider $\delta t$ sufficiently small with respect to the PU transmission duration. In this case, partial overlap can be neglected and the analysis can be simplified considering, either total overlap or no overlap, respectively. This approximation leads to a tractable performance analysis that matches very well with numerical results.

${ }^{10}$ The same approach can also be considered to account for shadow fading.
}

TABLE I

Evaluation of $P_{\text {TD }}$ FOR TST- $n m$ BASED ON (2a).

\begin{tabular}{c|c|c|}
\cline { 2 - 3 } & \multicolumn{2}{|c|}{ TST-nm } \\
\cline { 2 - 3 } & const. $\tau$ & random $\tau$ \\
\hline $\mathbb{P}\{\mathcal{S}\}$ & $(23)$ & $(38)$ \\
\hline $\mathbb{P}\{\mathcal{T}\}$ & $(24)$ & $(39)$ \\
\hline$P_{\mathrm{D}}$ & \multicolumn{2}{|c|}{ (13) or (15) } \\
\hline$P_{\mathrm{FA}}$ & \multicolumn{2}{|c|}{ (14) } \\
\hline
\end{tabular}

TABLE II

EVAluation of $P_{\text {TD }}$ FOR TST-S BASEd ON (2a).

\begin{tabular}{c|c|c|}
\cline { 2 - 3 } & \multicolumn{2}{|c|}{ TST-S } \\
\cline { 2 - 3 } & const. $\tau$ & random $\tau$ \\
\hline $\mathbb{P}\{\mathcal{S}\}$ & $(26),(29)$ & $(40),(42)$ \\
\hline $\mathbb{P}\{\mathcal{T}\}$ & $(27),(28),(30)$ & $(41),(28),(43)$ \\
\hline$P_{\mathrm{D}}$ & \multicolumn{2}{|c|}{$(13)$ or $(15)$} \\
\hline$P_{\mathrm{FA}}$ & \multicolumn{2}{|c|}{$(14)$} \\
\hline
\end{tabular}

\section{A. Temporal Detection over a Single Scan}

The temporal detection probability for the sensing tasks TST- $n m$ and TST-S can be derived from (2a) inserting the expressions of the probability of detection corresponding to the AWGN or fading scenario, i.e. (13) or (15), respectively, and the specific probabilities $\mathbb{P}\{\mathcal{S}\}$ and $\mathbb{P}\{\mathcal{T}\}$ related to the TST studied and the PU transmission statistic. Closedform expressions of $\mathbb{P}\left\{\mathcal{S}_{n, m}\right\}$ and $\mathbb{P}\left\{\mathcal{T}_{n, m}\right\}$ for TST- $n m$ and expressions for $\mathbb{P}\left\{\mathcal{S}_{m}\right\}$ and $\mathbb{P}\left\{\mathcal{T}_{m}\right\}$ for TST-S, are given in Section IV and $\mathrm{V}$ for the constant and random ON-period duration cases, respectively, considering both the transient phase and the steady-state regime. Tables I and II summarize the proper equations to be adopted in (2a) for the evaluation of the temporal detection probability.

\section{B. Temporal Detection over Multiple Scans}

In this section, we describe the temporal detection probability for the TST-M case, considering the OR rule for hard fusion and the EGC for soft fusion.

When the OR rule is applied, the temporal detection probability can be computed from (7) using the proper $P_{\mathrm{D}}$ (corresponding to the AWGN or fading case) and the closedform expressions of $P_{\mathrm{S}}(m)$ and $P_{\mathrm{T}}^{\mathrm{M}}$ that are presented in Section IV and $\mathrm{V}$ for the constant and random ON-period duration case, respectively. ${ }^{11}$ For convenience, in Table III we recap the equations to be adopted for TST-M with the OR rule.

For the soft fusion with EGC, we proceed, similarly to (11), denoting the received samples in the $m$ th sensing slot as $y_{j, m}=x_{j, m}+n_{j, m}$, described by a complex Gaussian r.v. with mean $x_{j, m}$ and variance $2 \sigma^{2}$. Note that in the $\overline{\mathcal{S}}$ hypothesis, i.e., when the PU transmission is not overlapped with the sensing interval, we have $x_{j, m}=0$ and thus $y_{j, m}$ has zero mean. With EGC, the decision statistic $\Lambda_{\mathrm{SF}}$ is given by

$$
\Lambda_{\mathrm{EGC}}=\sum_{m \in \Omega_{m}} \sum_{j=1}^{N_{s}} \frac{1}{2 \sigma^{2} N_{s}}\left|y_{j, m}\right|^{2}
$$

\footnotetext{
${ }^{11}$ Note that, adopting (15) in (7), we are implicitly assuming that the channel fading is constant among the different scans.
} 
TABLE III

EVALUATION OF $P_{\mathrm{TD}}^{\mathrm{FU}}$ FOR THE OR FUSION RULE WITH ED, BASED ON (7)

\begin{tabular}{c|c|c|}
\multirow{2}{*}{} & \multicolumn{2}{|c|}{ TST-M for ED-OR } \\
\cline { 2 - 3 } & const. $\tau$ & random $\tau$ \\
\hline $\mathbb{P}\{\mathcal{S}\}$ & $(26),(29)$ & $(40),(42)$ \\
\hline $\mathbb{P}\{\mathcal{T}\}$ & $(31),(33),(32)$ & $(44),(33),(45)$ \\
\hline$P_{\mathrm{D}}$ & \multicolumn{2}{|c|}{ (13) or (15) } \\
\hline$P_{\mathrm{FA}}$ & \multicolumn{2}{|c|}{$(14)$} \\
\hline
\end{tabular}

TABLE IV

EVALUATION OF $P_{\mathrm{TD}}^{\mathrm{FU}}$ FOR THE EGC FUSION RULE WITH ED, BASED ON (10).

\begin{tabular}{c|c|c|}
\multirow{2}{*}{} & \multicolumn{2}{|c|}{ TST-M for ED-EGC } \\
\cline { 2 - 3 } & const. $\tau$ & random $\tau$ \\
\hline $\mathbb{P}\{\mathcal{S}\}$ & $(29)$ & $(42)$ \\
\hline $\mathbb{P}\{\mathcal{T}\}$ & $(33),(32)$ & $(33),(45)$ \\
\hline $\mathbb{P}\left\{\Lambda_{\mathrm{SF}}>\xi \mid K=k\right\}$ & \multicolumn{2}{|c}{$(17)$} \\
\hline
\end{tabular}

Note that (16) is chi squared distributed with $2 N_{s} M$ degrees of freedom and non centrality parameter $\lambda_{\mathrm{EGC}}=K \rho .^{12}$ Thus for the ED-EGC strategy in AWGN the probability $\mathbb{P}\left\{\Lambda_{\mathrm{SF}}>\xi \mid K=k\right\}$ in (10) becomes

$$
\mathbb{P}\left\{\Lambda_{\mathrm{EGC}}>\xi \mid K=k\right\}=\mathcal{Q}_{N_{\mathrm{s}} M}\left(\sqrt{2 N_{\mathrm{s}} K \rho}, \sqrt{2 N_{\mathrm{s}} \xi}\right)
$$

where $\xi$ is the decision threshold. Note that, in the steady-state regime, the probability of overlap among PU transmissions and sensing intervals is constant $\forall m \in \Omega_{\mathrm{M}}$, and thus $K$ follows a binomial distribution. Therefore, we have $p_{k}=$ $\left(\begin{array}{c}M \\ k\end{array}\right) p^{k}(1-p)^{M-k}$ with $p=P_{\mathrm{S}} / P_{\mathrm{T}}^{\mathrm{M}}$, where the equations for $P_{\mathrm{S}}$ and $P_{\mathrm{T}}^{\mathrm{M}}$ can be obtained from Table IV. In case in which fading is considered, the average probability of detection can be computed, similarly to (15), averaging (17) with respect to the p.d.f. of $\rho$. When the PU is inactive, $\Lambda_{\mathrm{EGC}}$ is a central chi squared distributed r.v. with $2 N_{s} M$ degrees of freedom, and therefore the probability of false alarm can be written as

$$
P_{\mathrm{FA}}^{\mathrm{FU}}=\widetilde{\Gamma}\left(M N_{\mathrm{s}}, M N_{\mathrm{s}} \xi\right) .
$$

\section{Temporal Sensing Probability with Random ARrival AND CONSTANT Hold-Time}

In the previous section we derived the temporal detection probabilities for TST-mn, TST-S, and TST-M. All the closedform expressions obtained require the evaluation of the temporal sensing probabilities which depend on the PU temporal behavior and periodic sensing parameters. In this section, we calculate such probabilities when the PU has a temporal behavior characterized by a constant hold-time $\tau$.

In this case, the $n$th transmission from the PU occurs at time $t_{n}$ given by

$$
t_{n}=t_{0}+\sum_{i=1}^{n} \Delta_{i}+(n-1) \tau, \quad n \in \mathbb{N}
$$

where $t_{0}$ is an arbitrary time instant when the CR node starts to sense the spectrum, and assumed to be zero hereafter, without loss of generality. Since $\Delta_{i}$ are exponentially i.i.d. with parameter $\lambda$, the term $G_{n}=\sum_{i=1}^{n} \Delta_{i}$ follows an Erlang

\footnotetext{
${ }^{12}$ Here we assume that the SNR, $\rho$, is constant in the different sensing intervals.
}

distribution, denoted hereafter as $G_{n} \sim \operatorname{Erlang}(n, \lambda)$, with p.d.f. [30]

$$
f_{G_{n}}(x)=\frac{\lambda^{n} x^{n-1} \exp (-\lambda x)}{\Gamma(n)} u(x)
$$

where $u(x)$ is the unit step function, and the gamma function with integer parameter is $\Gamma(n)=(n-1)$ !. The start time for the $n$th transmission is then given by $t_{n}=G_{n}+(n-1) \tau$, and the corresponding p.d.f. is $f_{t_{n}}(x)=f_{G_{n}}(x-(n-1) \tau)$, i.e.,

$f_{t_{n}}(x)=\frac{\lambda^{n}(x-(n-1) \tau)^{n-1}}{\Gamma(n)} e^{-\lambda(x-(n-1) \tau)} u(x-(n-1) \tau)$.

From (21) the cumulative distribution function (c.d.f.) of $t_{n}$ is given by

$$
F_{t_{n}}(x)=\bar{\gamma}(n, \lambda(x-(n-1) \tau))
$$

where $\bar{\gamma}(a, z) \triangleq \gamma(a, z) u(z)$, and $\gamma(a, z) \quad=$ $\frac{1}{\Gamma(a)} \int_{0}^{z} t^{a-1} \exp (-t) d t$ is the incomplete gamma function.

\section{A. Probability of Sensing the $n$th Transmission During the $m$ th Scan}

With a constant $\mathrm{ON}$-period duration $\tau$, the $\mathrm{SU}$ senses the PU in the $m$ th scan if a PU transmission time falls within the interval $\left[m T_{w}-\delta t-\tau, m T_{w}\right)$. Hence, the probability corresponding to the event $\mathcal{S}_{n, m}$ is $P_{\mathrm{S}}^{(n)}(m)=\mathbb{P}\left\{\mathcal{S}_{n, m}\right\}=$ $\mathbb{P}\left\{m T_{w}-\delta t-\tau \leq t_{n}<m T_{w}\right\}$, and can be expressed in closed-form by (22) as

$$
\begin{aligned}
P_{\mathrm{S}}^{(n)}(m)=\bar{\gamma} & \left(n, \lambda\left(m T_{w}-(n-1) \tau\right)\right) \\
& -\bar{\gamma}\left(n, \lambda\left(m T_{w}-\delta t-n \tau\right)\right) .
\end{aligned}
$$

Similarly to (23), the probability that the $n$th PU transmission occurs within the $m$ th scan (the event $\mathcal{T}_{n, m}$ ) can be written as

$$
\begin{aligned}
P_{\mathrm{T}}^{(n)}(m)=\mathbb{P}\left\{\mathcal{T}_{n, m}\right\}= & \bar{\gamma}\left(n, \lambda\left(m T_{w}-(n-1) \tau\right)\right) \\
& -\bar{\gamma}\left(n, \lambda\left((m-1) T_{w}-n \tau\right)\right) .
\end{aligned}
$$

Note that $P_{\mathrm{S}}^{(n)}(m)$ in (23) and $P_{\mathrm{T}}^{(n)}(m)$ in (24) are zero when $n \geq n_{\max }$, where $n_{\max }=\left\lfloor m T_{w} / \tau-1\right\rfloor$ is the maximum number of possible PU transmissions up to the $m$ th scan. ${ }^{13}$ According to Section II-B, the temporal sensing probability $P_{\mathrm{TS}}^{(n)}(m)=\mathbb{P}\left\{\mathcal{S}_{n, m}\right\} / \mathbb{P}\left\{\mathcal{T}_{n, m}\right\}$ can be written in closedform as

$$
\begin{aligned}
& P_{\mathrm{TS}}^{(n)}(m)= \\
& \quad \frac{\bar{\gamma}\left(n, \lambda\left(m T_{w}-(n-1) \tau\right)\right)-\bar{\gamma}\left(n, \lambda\left(m T_{w}-\delta t-n \tau\right)\right)}{\bar{\gamma}\left(n, \lambda\left(m T_{w}-(n-1) \tau\right)\right)-\bar{\gamma}\left(n, \lambda\left((m-1) T_{w}-n \tau\right)\right)} .
\end{aligned}
$$

From the above equation we observe that for continuous sensing, i.e., as $\delta t$ approaches $T_{w}$, the temporal sensing probability becomes one, as expected.

\footnotetext{
${ }^{13}\lfloor x\rfloor$ stands for the largest integer not greater than $x$.
} 


\section{B. Probability of Sensing the PU During a Single Scan}

1) Transient phase: The probability $P_{S}(m)=\mathbb{P}\left\{\mathcal{S}_{m}\right\}=$ $\mathbb{P}\left\{\exists t_{n} \in\left[m T_{w}-\delta t-\tau, m T_{w}\right]\right\}$ is derived in Appendix-A, and can be expressed in closed form as

$$
P_{\mathrm{S}}(m)=1-\sum_{n=0}^{n_{\max }} \frac{\lambda^{n}\left(m T_{w}-\delta t-n \tau\right)^{n}}{n !} e^{-\lambda\left(m T_{w}-n \tau\right)} .
$$

For the derivation of $P_{\mathrm{T}}(m)=\mathbb{P}\left\{\mathcal{T}_{m}\right\}=$ $\mathbb{P}\left\{\exists t_{n} \in\left[(m-1) T_{w}-\tau, m T_{w}\right]\right\}$, for $m>1$, we can adopt the same approach, that leads to the expression

$$
P_{\mathrm{T}}(m)=1-\sum_{n=0}^{n_{\max }} \frac{\lambda^{n}\left((m-1) T_{w}-n \tau\right)^{n}}{n !} e^{-\lambda\left(m T_{w}-n \tau\right)}
$$

while for $m=1$ it is simply

$$
P_{\mathrm{T}}(1)=\mathbb{P}\left\{0 \leq t_{1} \leq T_{w}\right\}=\bar{\gamma}\left(1, \lambda T_{w}\right) .
$$

2) Steady-state regime: In the steady-state scenario, the probability to capture any PU transmission in a sensing interval, $P_{\mathrm{S}}$, can be derived considering that it can be written as the sum of two terms: the first one is the probability of having a transmission within the sensing interval conditioned to the absence of a transmission at the beginning of the sensing interval, and the second is the probability of having a transmission present at the beginning of the sensing interval. Hence, ${ }^{14}$

$$
P_{\mathrm{S}}=1-e^{-\lambda \delta t} \frac{\lambda^{-1}}{\lambda^{-1}+\tau}=1-\frac{e^{-\lambda \delta t}}{1+\lambda \tau} .
$$

With the same approach, the probability to have any PU transmission in a scan is

$$
P_{\mathrm{T}}=1-\frac{e^{-\lambda T_{w}}}{1+\lambda \tau} .
$$

In Fig. 3 an example of $P_{S}(m)$ as a function of $m$ is reported. As we can see, (26) matches the simulations and, after the transient phase, it converges to (29).

\section{Temporal Sensing Probability over Multiple Scans}

In Section III-B, we derived the temporal detection probability over multiple scans considering independence between sensing performed in the $M$ sensing intervals. Considering the OR fusion rule, to apply (8) it is necessary to adopt (26) in the transient phase, and to adopt (29) for the steady-state regime. Denoting with $m_{\min }$ and $m_{\max }$ the minimum and the maximum indexes in $\Omega_{M}$, respectively, the probability that a transmission occurs within $M$ scans can be written using the results obtained in Appendix-A, and is given by

$$
\begin{aligned}
P_{\mathrm{T}}^{\mathrm{M}} & =\mathbb{P}\left\{\mathcal{T}_{M}\right\}=\mathbb{P}\left\{\exists t_{n} \in\left[\left(m_{\min }-1\right) T_{w}-\tau, m_{\max } T_{w}\right]\right\} \\
& =1-\sum_{n=0}^{n_{\max }} \frac{\lambda^{n}\left(\left(m_{\min }-1\right) T_{w}-n \tau\right)^{n}}{n !} e^{-\lambda\left(m_{\max } T_{w}-n \tau\right)}
\end{aligned}
$$

\footnotetext{
${ }^{14}$ Note that in the steady-state regime the temporal sensing probabilities are independent on the particular sensing interval considered, and thus the index $m$ is omitted.
}

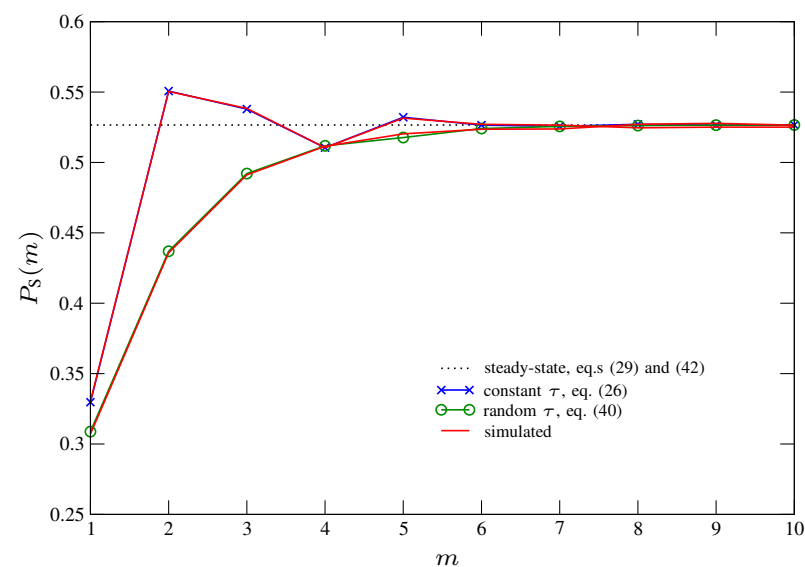

Fig. 3. Temporal sensing probability $P_{\mathrm{S}}(m)$ as a function of $m$ for $T_{w}=$ $0.5 \mathrm{~s}, \delta t=0.2 \mathrm{~s}, \lambda=0.8 \mathrm{~s}^{-1}$ and $\tau=\bar{\tau}=1 \mathrm{~s}$. Comparison between simulations and the closed-form expressions (26) and (40) is also given.

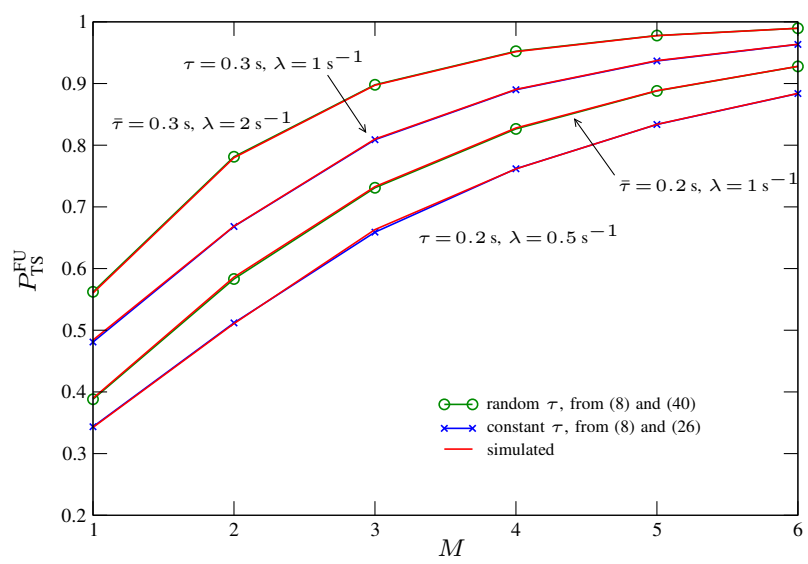

Fig. 4. Temporal sensing probability for multiple scans, with the OR rule, as a function of the number of scans considered, $M$, with $\delta t=0.1 \mathrm{~s}, T_{w}=1 \mathrm{~s}$. Comparison between simulations and the closed-form expressions (8) with (26) and (40) is also given.

for the transient phase, and by

$$
P_{\mathrm{T}}^{\mathrm{M}}=1-\frac{e^{-\lambda M T_{w}}}{1+\lambda \tau}
$$

for the steady-state regime. If we consider the first $M$ sensing periods, (31) reduces to

$$
P_{\mathrm{T}}^{\mathrm{M}}=\bar{\gamma}\left(1, \lambda M T_{w}\right)
$$

To prove the validity of the derived temporal sensing probability, in Fig. 4 we compare theoretical curves obtained from (8) with (26) and simulations. As can be seen, despite the approximations introduced, the theoretical probabilities match very well the simulated curves.

Considering the EGC rule, assuming the steady-state regime, $P_{\mathrm{TD}}^{\mathrm{FU}}$ is given in (10), where $p$ is computed using (29) for $P_{\mathrm{S}}$ and (32) or (33) for $P_{\mathrm{T}}^{\mathrm{M}}$, and the conditional probability is given by (17).

\section{Temporal Sensing Probability with Random ARRIVAL AND RANDOM HOLD-TIME}

In this section we derive the temporal sensing probabilities for the three TSTs considering an exponentially distributed 
ON-period duration. In this case, the $n$th PU transmission occurs at time $t_{n}$ given by

$$
t_{n}=\sum_{i=1}^{n} \Delta_{i}+\sum_{i=1}^{n-1} \tau_{i}=G_{n}+H_{n}, \quad n \in \mathbb{N}
$$

where $H_{n}=\sum_{i=1}^{n-1} \tau_{i} \sim \operatorname{Erlang}(n-1, \mu)$. Hence, $t_{n}$ is the sum of two Erlang distributed r.v.s with parameters $\lambda$ and $\mu$, respectively.

Using the results derived in [35], the complementary c.d.f. of $t_{n}$ can be expressed as a linear combination of incomplete gamma functions as

$$
\begin{aligned}
Q_{t_{n}}(x) & =1-F_{t_{n}}(x) \\
& =\sum_{p=1}^{n} c_{1 p} \bar{\Gamma}(n-p+1, \lambda x)+\sum_{q=1}^{n-1} c_{2 q} \bar{\Gamma}(n-q, \mu x)
\end{aligned}
$$

where $\bar{\Gamma}(a, b)=1-\bar{\gamma}(a, b)$ is the normalised upper incomplete Gamma function, and

$$
\begin{aligned}
c_{1 p} & =(-1)^{p-1} \frac{\lambda^{p-1} \mu^{n-1}}{(\mu-\lambda)^{n+p-2}}\left(\begin{array}{c}
n+p-3 \\
p-1
\end{array}\right) \\
c_{2 q} & =(-1)^{q-1} \frac{\lambda^{n} \mu^{q-1}}{(\lambda-\mu)^{n+q-1}}\left(\begin{array}{c}
n+q-2 \\
q-1
\end{array}\right) .
\end{aligned}
$$

\section{A. Probability of Sensing the nth Transmission During the mth Scan}

The probability that the $n$th transmission falls into the $m$ th sensing interval can be computed as $P_{\mathrm{S}}^{(n)}(m)=$ $\mathbb{E}_{\tau}\left\{\mathbb{P}\left\{\left(m T_{w}-\delta t-\tau\right) \leq t_{n}<m T_{w}\right\}\right\}$. From (35) and the integral expressions derived in Appendix-B, we can express $P_{\mathrm{S}}^{(n)}(m)$ in closed-form as in (38).

Similarly to (38), the probability $P_{\mathrm{T}}^{(n)}(m)$ can be expressed as in (39). From (38) and (39) we obtain the temporal sensing probability $P_{\mathrm{TS}}^{(n)}(m)$ which can be used in (2a) to calculate $P_{\mathrm{TD}}^{(n)}(m)$.

\section{B. Probability of Sensing the PU During a Single Scan}

1) Transient phase: Similarly to (26) and (27), the probabilities $P_{\mathrm{S}}(m)$ and $P_{\mathrm{T}}(m)$ can be derived, as detailed in Appendix-A, as

$$
\begin{aligned}
P_{\mathrm{S}}(m)= & -e^{-\lambda m T_{w}} \\
& -\sum_{n=0}^{\infty} \mathbb{P}\left\{t_{n+1}>m T_{w}, t_{n}<m T_{w}-\delta t-\tau\right\} \\
P_{\mathrm{T}}(m)= & -e^{-\lambda m T_{w}} \\
& -\sum_{n=0}^{\infty} \mathbb{P}\left\{t_{n+1}>m T_{w}, t_{n}<(m-1) T_{w}-\tau\right\}
\end{aligned}
$$

that can be expressed in closed-form using (52) and (60). In practice, the infinite summations in (40) and (41) can be truncated to a finite number $n_{\max }^{*}$, considering that, as numerical simulations confirm, the arguments rapidly vanish with $n$. Note that, for the $m=1$ case, $P_{\mathrm{T}}(m)$ is given by (28).

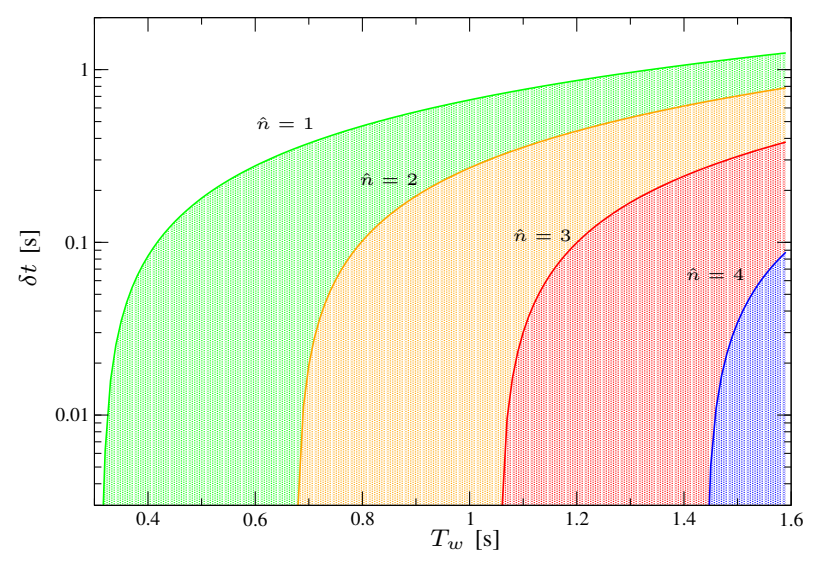

Fig. 5. Minimum sensing duration $\delta t$ as a function of $T_{w}$ for detecting the $n$th transmission with $P_{\mathrm{TS}}^{\mathrm{DES}}=0.95$. The shaded areas below the curves indicate the regions where detection requirement, $P_{\mathrm{TS}}>P_{\mathrm{TS}}^{\mathrm{DES}}$, is not satisfied.

2) Steady-state regime: In the steady-state scenario, it is possible to derive the sensing probability, $P_{\mathrm{S}}$, and the transmission probability, $P_{\mathrm{T}}$, following the same approach presented in Section IV-B, which leads to ${ }^{15}$

$$
\begin{aligned}
& P_{\mathrm{S}}=1-\frac{e^{-\lambda \delta t}}{1+\lambda \bar{\tau}} \\
& P_{\mathrm{T}}=1-\frac{e^{-\lambda T_{w}}}{1+\lambda \bar{\tau}} .
\end{aligned}
$$

In Fig. 3 we can see an example of $P_{S}(m)$ as a function of $m$. The figure confirms also that (40) matches the simulated curve and that after the transient phase it converges to (42).

\section{Probability of Detection over Multiple Scans}

To apply (8) it is necessary to adopt (40) to derive the performance in the transient phase, or (42) for the steady-state regime. Considering the generic set $\Omega_{M}$, similarly to (31) and (41), the probability $\mathbb{P}\left\{\mathcal{T}_{M}\right\}$ is given by

$$
\begin{aligned}
P_{\mathrm{T}}^{\mathrm{M}}=1 & -e^{-\lambda m_{\max } T_{w}} \\
& -\sum_{n=0}^{\infty} \mathbb{P}\left\{t_{n+1}>m_{\max } T_{w}, t_{n}<\left(m_{\min }-1\right) T_{w}-\tau\right\}
\end{aligned}
$$

while considering the first $M$ sensing periods it is given by (33). as

In the steady-state regime, $\mathbb{P}\left\{\mathcal{T}_{M}\right\}$ can be easily expressed

$$
P_{\mathrm{T}}^{\mathrm{M}}=1-\frac{e^{-\lambda M T_{w}}}{1+\lambda \bar{\tau}} .
$$

Note that if we consider the first $M$ scans, $\mathbb{P}\left\{\mathcal{T}_{M}\right\}$ is given by (33).

Considering the EGC rule, assuming the steady-state regime, $P_{\mathrm{TD}}^{\mathrm{FU}}$ is given in (10), where $p$ is computed using (42) for $P_{\mathrm{S}}$ and (45) or (33) for $P_{\mathrm{T}}^{\mathrm{M}}$, and the conditional probability is given by (17).

\footnotetext{
${ }^{15}$ Note that in the steady-state regime, the two probabilities (42) and (43) have the same form as (29) and (30) where now $\bar{\tau}$ appears in place of $\tau$.
} 


$$
\begin{aligned}
& P_{\mathrm{S}}^{(n)}(m)=\int_{0}^{\infty} Q_{t_{n}}\left(m T_{w}-\delta t-\tau\right) \frac{1}{\bar{\tau}} \exp \left(-\frac{\tau}{\bar{\tau}}\right) d \tau-Q_{t_{n}}\left(m T_{w}\right) \\
& =\sum_{p=1}^{n} c_{1 p}\left(\bar{\gamma}\left(n-p+1, \lambda m T_{w}\right)-\bar{\gamma}\left(n-p+1, \lambda\left(m T_{w}-\delta t\right)\right)\right. \\
& \left.+\gamma\left(n-p+1, \lambda\left(m T_{w}-\delta t\right)\left(1-\frac{\mu}{\lambda}\right)\right)\left(\frac{\lambda}{\lambda-\mu}\right)^{n-p+1} \exp \left(-\mu\left(m T_{w}-\delta t\right)\right)\right) \\
& +\sum_{q=1}^{n-1} c_{2 q}\left(\bar{\gamma}\left(n-q, \mu m T_{w}\right)-\bar{\gamma}\left(n-q, \mu\left(m T_{w}-\delta t\right)\right)\right. \\
& \left.+\frac{\left(\mu\left(m T_{w}-\delta t\right)\right)^{n-q}}{(n-q) !} \exp \left(-\mu\left(m T_{w}-\delta t\right)\right)\right) . \\
& P_{\mathrm{T}}^{(n)}(m)=\int_{0}^{\infty} Q_{t_{n}}\left((m-1) T_{w}-\tau\right) \frac{1}{\bar{\tau}} \exp \left(-\frac{\tau}{\bar{\tau}}\right) d \tau-Q_{t_{n}}\left(m T_{w}\right) \\
& =\sum_{p=1}^{n} c_{1 p}\left(\bar{\gamma}\left(n-p+1, \lambda m T_{w}\right)-\bar{\gamma}\left(n-p+1, \lambda\left((m-1) T_{w}\right)\right)\right. \\
& \left.+\gamma\left(n-p+1, \lambda\left((m-1) T_{w}\right)\left(1-\frac{\mu}{\lambda}\right)\right)\left(\frac{\lambda}{\lambda-\mu}\right)^{n-p+1} \exp \left(-\mu\left((m-1) T_{w}\right)\right)\right) \\
& +\sum_{q=1}^{n-1} c_{2 q}\left(\bar{\gamma}\left(n-q, \mu m T_{w}\right)-\bar{\gamma}\left(n-q, \mu\left((m-1) T_{w}\right)\right)\right. \\
& \left.+\frac{\left(\mu\left((m-1) T_{w}\right)\right)^{n-q}}{(n-q) !} \exp \left(-\mu\left((m-1) T_{w}\right)\right)\right) .
\end{aligned}
$$

\section{Vi. Design Strategies and Results}

In this section we present some design strategies for periodic sensing based on the derived temporal detection framework. We start addressing the design of the sensing duration $\delta t$ and the sensing period $T_{w}$. We then derive the complementary receiver operating characteristic (C-ROC) curves, and finally we provide a case study for periodic sensing in which the PU is represented by a Wi-Fi system.

\section{A. Minimum Sensing Time to Guarantee a given Temporal Detection Probability}

Considering a PU adopting a packet-based transmission, from the regulatory point of view as well as from the PU perspective, it is important to quantify or control how many PU packets are interfered by the SU. For instance, assume that the PU may tolerate $\hat{n}-1$ undetected transmissions, and that for $n \geq \hat{n}$ all transmissions are interfered with a probability which cannot exceed $P_{\mathrm{I}}^{\mathrm{max}}$, named maximum probability of interference. A simple metric for quantifying the maximum interference to the PU is the missed detection probability, i.e. $P_{\mathrm{I}}^{\max }=1-P_{\mathrm{TD}}^{\mathrm{DES}}$ where $P_{\mathrm{TD}}^{\mathrm{DES}}$ is the minimum desired probability of detection. ${ }^{16}$ Then, considering $m=1$, and, for instance, a PU with fixed packet duration, we are interested in designing the minimum sensing duration $\delta t$. If, for example,

\footnotetext{
${ }^{16}$ Note that the probability of missed detection is a worst case probability of interference, derived assuming that the SU transmit whenever it declares the channel free.
}

we consider the situation in which the SNR is high, the temporal detection probability reduces to the temporal sensing probability $\left(P_{\mathrm{TD}} \approx P_{\mathrm{TS}}\right.$ ), hence from (25) it is possible to derive the following inequality

$$
\frac{\bar{\gamma}\left(\hat{n}, \lambda\left(T_{w}-\delta t-\hat{n} \tau\right)\right)}{\bar{\gamma}\left(\hat{n}, \lambda\left(T_{w}-(\hat{n}-1) \tau\right)\right)} \leq 1-P_{\mathrm{TD}}^{\mathrm{DES}}
$$

which can be inverted to derive ${ }^{17}$

$$
\begin{aligned}
\delta t \geq & T_{w}-\hat{n} \tau \\
& +\frac{1}{\lambda} \bar{\gamma}^{-1}\left(\hat{n},\left(1-P_{\mathrm{TD}}^{\mathrm{DES}}\right) \bar{\gamma}\left(\hat{n}, \lambda\left(T_{w}-(\hat{n}-1) \tau\right)\right)\right) .
\end{aligned}
$$

The expression (47) allows the design of $\delta t$, given the PU transmission parameters, $\lambda, \tau$, and the scanning period $T_{w}$ to guarantee $P_{\mathrm{TD}}^{\mathrm{DES}}$. As an example, in Fig. 5 we show the minimum $\delta t$ required to satisfy $P_{\mathrm{TD}}^{\mathrm{DES}}=0.95$ as a function of $T_{w}$ for different values of $\tilde{n}$. The shaded areas denote the regions where the interference probability constraint is not satisfied. Note that Fig. 5 can be also used for the design of the sensing scan duration $T_{w}$, chosen a particular value of $\delta t$. Similar design strategies can be derived for the random holdtime case and/or considering the presence of noise through the corresponding expressions for $P_{\mathrm{TD}}$.

\footnotetext{
${ }^{17}$ If $\bar{\gamma}(a, z)=w$, then the inverse lower incomplete gamma function is defined as $z=\bar{\gamma}^{-1}(a, w)$. Note that this inverse expression can be easily computed using standard mathematical software.
} 


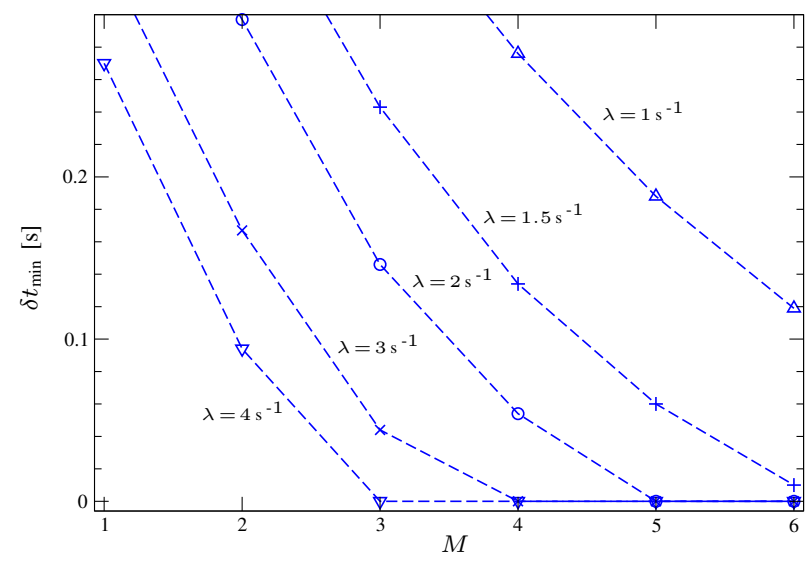

Fig. 6. Minimum sensing duration $\delta t$ required to reach a target $P_{\mathrm{TD}}^{\mathrm{DES}}=0.9$ with $\tau=0.3 \mathrm{~s}$ and $T_{w}=1 \mathrm{~s}$ for different values of $\lambda$.

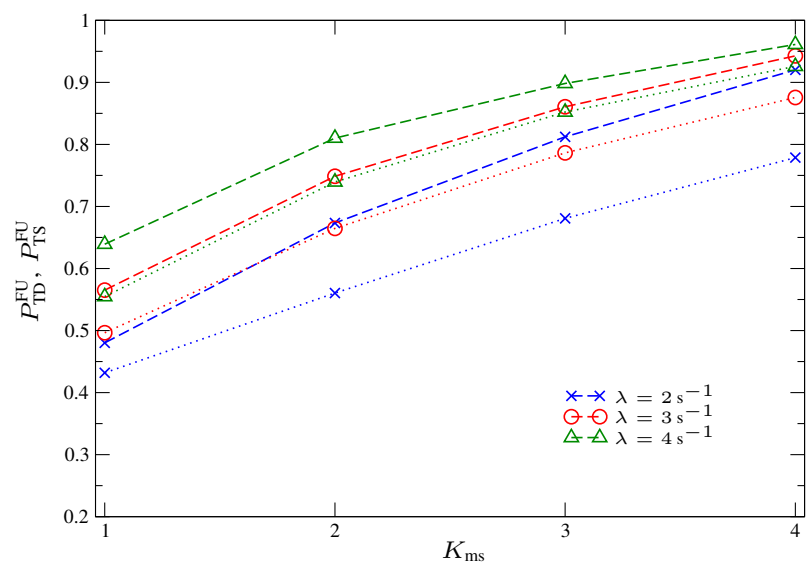

Fig. 7. $P_{\mathrm{TD}}^{\mathrm{FU}}$ (dotted lines) and $P_{\mathrm{TS}}^{\mathrm{FU}}$ (dashed lines) as a function of the number of mini-slots $K_{\mathrm{ms}}$ with constant and random PU hold-time $\tau=\bar{\tau}=0.2 \mathrm{~s}$, sensing period $T_{w}=1 \mathrm{~s}$, sensing duration $\delta t=0.1 \mathrm{~s}$, and $N_{\mathrm{s}}=84$. For $P_{\mathrm{TD}}^{\mathrm{FU}}$ an ED in the presence of AWGN with $\rho=15 \mathrm{~dB}$, and with a decision threshold set to guarantee a $P_{\mathrm{FA}}^{\mathrm{FU}}=0.1$, is considered.

The design of the sensing time required can be also performed when multiple scans are considered. In this case, by using equation (7), the minimum sensing interval duration, $\delta t_{\min }$, can be found as

$$
\delta t_{\min }=\min \left\{\delta t \mid P_{\mathrm{TD}}^{\mathrm{FU}} \geq P_{\mathrm{TD}}^{\mathrm{DES}}\right\} .
$$

For example, considering the OR fusion rule, in Fig. 6 we show the minimum $\delta t_{\min }$ as a function of the number of scans $M$ with $P_{\mathrm{TD}}^{\mathrm{DES}}=0.9, \tau=0.3 \mathrm{~s}$ and $T_{w}=1 \mathrm{~s}$, for different values of $\lambda$, when $\tau$ is constant and $\rho \rightarrow \infty$. As can be seen, an increase of $\lambda$, corresponding to a decrease of the OFF-period duration, requires shorter sensing durations to guarantee the same performance.

\section{B. Periodic Sensing by means of Mini-slots}

Assuming that the time dedicated to sensing for each scan is $d$, an interesting aspect to investigate if it is better to adopt a single sensing interval of duration $\delta t=d$ or to divide it into $K_{\mathrm{ms}}$ shorter periods, called mini-slots, equally spaced in the scan period by $\left(T_{w}-d\right) / K_{\mathrm{ms}}$ and each with a sensing window of duration $\delta t=d / K_{\mathrm{ms}}$. In Fig. 7 we show the

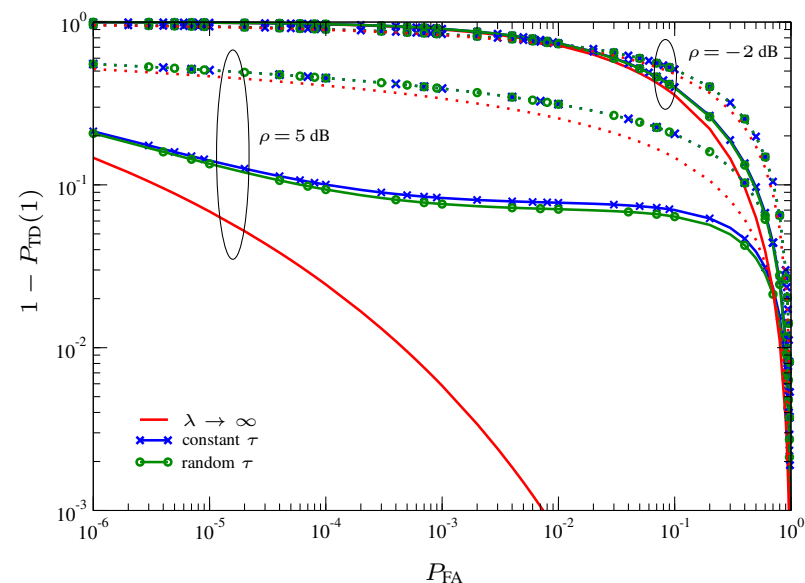

Fig. 8. Complementary ROC curves for various SNRs $\rho$ in AWGN (continuous lines) and Rayleigh fading (dotted lines) scenarios, with $T_{w}=1 \mathrm{~s}$, $\delta t=0.1 \mathrm{~s}, \bar{\tau}=\tau=0.2 \mathrm{~s}, N_{\mathrm{s}}=10$ and $\lambda=8 \mathrm{~s}^{-1}$.

$P_{\mathrm{TS}}^{\mathrm{FU}}$ relative to $K_{\mathrm{ms}}$ mini-slots when the OR rule is applied, for different values of $\lambda$, whit random $\tau$. It is clear that the adoption of multiple shorter sensing windows within a scan period provides an higher sensing probability. In the noisy case we plot $P_{\mathrm{TD}}^{\mathrm{FU}}$ which confirms the same behavior. This fact is explained by the ability of mini-slots to counteract the effect of non-continuous PU transmission, increasing the chance to have an overlap between a PU transmission and the sensing period.

\section{Receiver Operating Characteristics for Periodic Sensing}

In this section, we show some examples of ROC for temporal detection. We choose in particular to adopt the C-ROC curves to highlight the probability $1-P_{\mathrm{TD}}$ which, as discussed above, can be interpreted as a probability of interfering the PU. Fig. 8 depicts the C-ROC curves varying the SNR for AWGN and Rayleigh fading scenarios. From the figure we observe that for high values of $\rho$ the PU temporal behavior dominates the detection performance, which is poor compared to the scenario with a continuous PU transmission (i.e. $\lambda \rightarrow \infty$ ). This confirms that the performance of periodic sensing are severely degraded by the PU temporal behavior. For low SNR, instead, the dependence on $\lambda$ is smaller, and thus the noise is the dominant effect in the detection performance. Also note that the presence of Rayleigh fading results in a strong decrease in the detection probability. In this case the effects of fading is dominant with respect to the PU temporal behavior. Fig. 9 shows the C-ROC curves for multiple scans based on the OR rule, corresponding to equation (7) and (9), and the EGC approach described by (17). As expected, the detection performance improves with increasing $M$. Note, in particular, that soft fusion EGC always provides better performance with respect to the hard fusion based on the OR rule, especially for low $P_{\mathrm{FA}}$. This result suggests that soft fusion strategies should be preferred to hard fusion approaches.

In all cases, and for the parameters considered, the performance with random and constant hold-time, with $\tau=\bar{\tau}$, are very similar, indicating a small dependence of the hold-time statistic on the SU detection performance. 


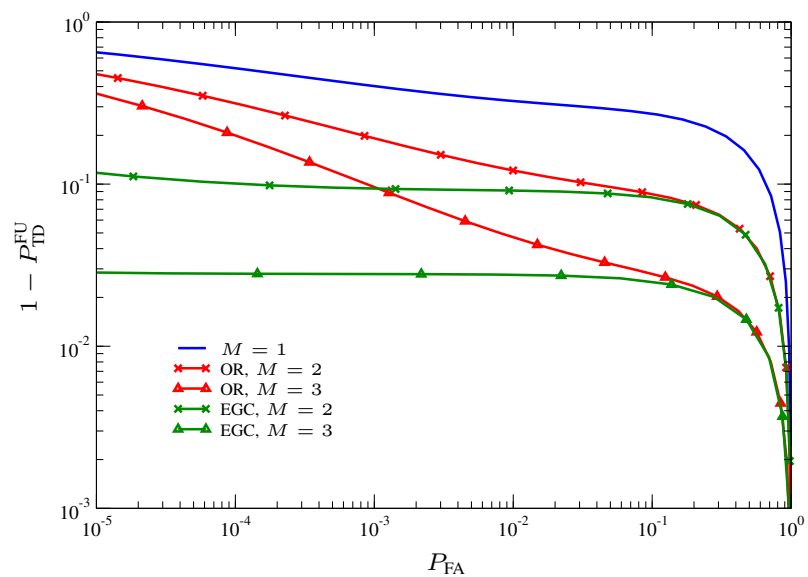

Fig. 9. Complementary ROC curves for the TST-M, considering ED with $\mathrm{OR}$ and EGC combining. An AWGN scenario with $\tau=0.2 \mathrm{~s}, \lambda=5 \mathrm{~s}^{-1}$, $\delta t=0.1 \mathrm{~s}, T_{w}=1 \mathrm{~s}$, and $N_{\mathrm{s}}=10$, and $\rho=3 \mathrm{~dB}$ have been considered.

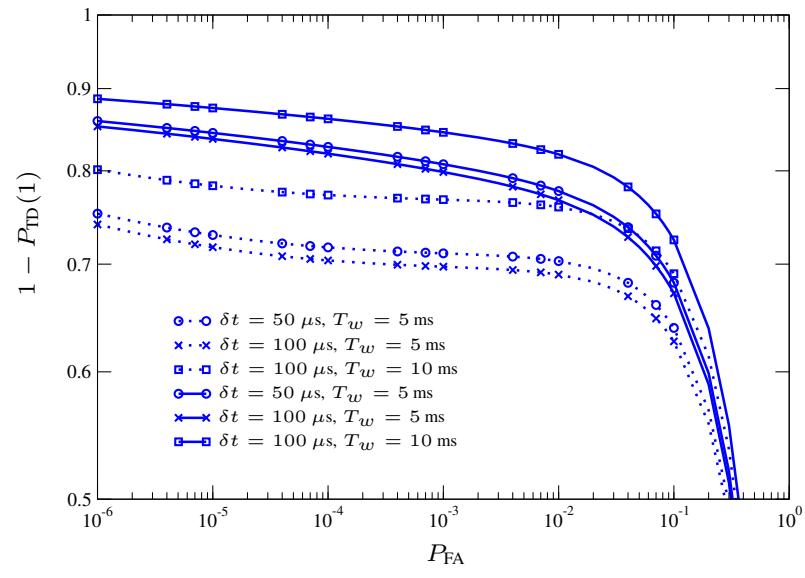

Fig. 10. Complementary ROC curves in AWGN (continuous lines) and Rayleigh fading (dotted lines) scenarios, with $\bar{\tau}=1 \mathrm{~ms}, \bar{\Delta}=1 / \lambda=4.2 \mathrm{~ms}$, $N_{\mathrm{s}}=10$, and $\rho=5 \mathrm{~dB}$. The PU parameters adopted are typical for Wi-Fi signals.

\section{Case study: detection of a Wi-Fi signal}

As a case study we propose the analysis of periodic sensing of a Wi-Fi signal considering the TST-S for the first sensing scan for different choices of $\delta t$ and $T_{w}$. In order to consider a realistic scenario, we adopt the measured parameter values presented in [13], [14]. In Fig. 10 we show the C-ROC for the AWGN and Rayleigh fading cases, considering $\tau=\bar{\tau}=1 \mathrm{~ms}$ and $\bar{\Delta}=4.2 \mathrm{~ms}$, typical of a VoIP application [13]. In [14] it has been shown that the statistic of the idle time noticeably depends on the amount of traffic, which also impacts the temporal detection probability. In Fig. 11 we show the detection performance considering $\bar{\Delta}=2.45,4.2$ and $10 \mathrm{~ms}$, which corresponds to the traffic scenarios with 100,75 and 25 pkts/s [14].

A second aspect investigated is the evaluation of the latency of periodic sensing, defined as the minimum number of PU transmissions for which the probability of interference is below $P_{\mathrm{I}}^{\max }$. This number, denoted with $\widetilde{n}$, is given by

$$
\tilde{n} \triangleq \arg \min _{n}\left\{P_{\mathrm{I}}=1-\frac{P_{\mathrm{S}}^{(n)}(1)}{P_{\mathrm{T}}^{(n)}(1)} \mid P_{\mathrm{I}} \leq P_{\mathrm{I}}^{\max }\right\} \text {. }
$$

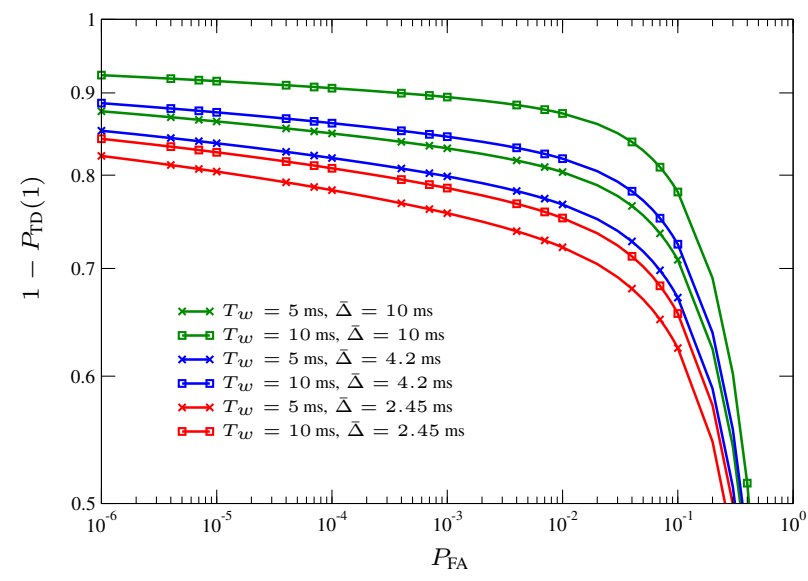

Fig. 11. Complementary ROC curves Rayleigh fading scenarios, with $\delta t=$ $100 \mu \mathrm{s}, \bar{\tau}=1 \mathrm{~ms}, N_{\mathrm{s}}=10$, and $\rho=5 \mathrm{~dB}$. The PU parameters adopted are typical for Wi-Fi signals and VoIP-like traffic.

TABLE V

EXAMPLES OF $\tilde{n}$ AND $R$ FOR THE WI-FI SIGNAL DETECTION CONSIDERED IN FIG. 10. $P_{\mathrm{TD}}^{\mathrm{DES}}=0.9$ AND $P_{\mathrm{FA}}^{\mathrm{DES}}=0.1$.

\begin{tabular}{|c|c|c|c|}
\hline & $\delta t=50 \mu \mathrm{s}$ & $\delta t=100 \mu \mathrm{s}$ & $\delta t=100 \mu \mathrm{s}$ \\
& $T_{w}=5 \mathrm{~ms}$ & $T_{w}=5 \mathrm{~ms}$ & $T_{w}=10 \mathrm{~ms}$ \\
\hline$\widetilde{n}$ & 4 & 4 & 8 \\
\hline$R$ & 0.696 & 0.676 & 0.752 \\
\hline
\end{tabular}

Note that when $n<\widetilde{n}$, the constraint on $P_{\mathrm{I}}$ is not guaranteed, and thus the sensing process requires at least $\tilde{n}$ transmissions to provide the required performance, i.e., $P_{\mathrm{TD}}>P_{\mathrm{TD}}^{\mathrm{DES}}$.

Finally, it is interesting to calculate simple metrics for quantifying the impact of periodic sensing on the performance of the SU, such as, for example, the maximum SU rate. We adopt, in particular, the maximum normalised achievable throughput, considered in [20], [37], [38], defined as $R=\frac{T_{w}-\delta t}{T_{w}} P_{\mathrm{TX}}$, where $P_{\mathrm{TX}}$ is the maximum $\mathrm{SU}$ transmission probability. In accordance with [37], we assume that the SU successfully transmits every time the sensing task decides for the absence of the PU, and thus we have

$$
R=\frac{T_{w}-\delta t}{T_{w}}\left(1-P_{\mathrm{TD}}\right)
$$

In Table $\mathrm{V}$ we report some numerical examples of $\tilde{n}$ and $R$, corresponding to the scenario considered in Fig. 10. As can be seen, an increase in $T_{w}$ from $5 \mathrm{~ms}$ to $10 \mathrm{~ms}$ corresponds to an increase in the latency and in the maximum SU rate. These results reveal the trade-off between the PU protection and the SU throughput.

\section{CONCLUSIONS}

In this paper we presented a general analytical framework for the study of the detection performance in CR contexts, taking into account, in particular, the presence of non-continuous PU transmissions and the adoption of periodic spectrum sensing. The analysis allows to study the sensing task in its entirety, but at the same time is able to separate the effects of detection impairments, such as noise, fading, and non-continuous PU transmissions, and to show their respective impact on the overall performance. Numerical results prove that the PU temporal behavior has a very strong impact on detection 
performance. Moreover, the relationship among arrival rate, occupancy duration, sensing period and sensing duration are revealed. The analysis is general enough to describe several practical scenarios considering both scan-by-scan sensing as well as sensing based on multiple scans. As a case study, the energy detector was chosen to derive numerical results. Based on the analysis developed, we then proposed some design strategies to set up periodic sensing parameters in different scenarios.

\section{APPENDIX A}

Constant $\tau$ case: computation of $\mathbb{P}\left\{\exists t_{n} \in[a, b]\right\}$

With the notation $\mathbb{P}\left\{\exists t_{n} \in[a, b]\right\}$ we indicate the probability that at least one transmission, represented by its starting time $t_{n}, \forall n \in \mathbb{N}$, falls in the interval $(a, b)$. This probability is computed in this section considering that $t_{n}$ is distributed as in (21), where $a$ and $b$ are constants. Note that, given the definition in (19), we can write

$$
\begin{aligned}
\mathbb{P}\left\{\exists t_{n} \in[a, b]\right\} & =1-\mathbb{P}\left\{\nexists t_{n} \in[a, b]\right\} \\
& =1-\mathbb{P}\left\{t_{1}>b \text { or } \bigcup_{n=1}^{n_{\max }}\left\{t_{n+1}>b, t_{n}<a\right\}\right\} \\
& =1-\mathbb{P}\left\{t_{1}>b\right\}-\sum_{n=1}^{n_{\max }} \mathbb{P}\left\{t_{n+1}>b, t_{n}<a\right\}
\end{aligned}
$$

where the last equality is given by the union of mutually exclusive events and $n_{\max }=\lfloor b / \tau-1\rfloor$. Considering that $t_{n+1}=t_{n}+\Delta_{n+1}+\tau$, we have $\mathbb{P}\left\{t_{n+1}>b, t_{n}<a\right\}=$ $\mathbb{P}\left\{\Delta_{n+1}>b-\tau-t_{n}, t_{n}<a\right\}$, and, given that $t_{n}$ and $\Delta_{n+1}$ are independent r.v.s, we can write

$$
\begin{aligned}
\mathbb{P} & \left\{\Delta_{n+1}>b-\tau-t_{n}, t_{n}<a\right\} \\
& =\int_{t_{n}<a} \mathbb{P}\left\{\Delta_{n+1}>b-\tau-t\right\} f_{t_{n}}(t) d t \\
& =\int_{t_{n}<a} e^{-\lambda(b-\tau-t)} f_{t_{n}}(t) d t \\
& =\frac{\lambda^{n}(a-(n-1) \tau)^{n}}{n !} e^{-\lambda(b-n \tau)} .
\end{aligned}
$$

Considering that

$$
\mathbb{P}\left\{t_{1}>b\right\}=\exp (-\lambda b)
$$

we can express (51) as

$$
\mathbb{P}\left\{\exists t_{n} \in[a, b]\right\}=1-\sum_{n=0}^{n_{\max }} \frac{\lambda^{n}(a-(n-1) \tau)^{n}}{n !} e^{-\lambda(b-n \tau)} .
$$

Random $\tau$ case: computation of $\mathbb{P}\left\{\exists t_{n} \in[a-\tau, b]\right\}$.

For the random hold time $\tau$ case, in this section we compute the probability $\mathbb{P}\left\{\exists t_{n} \in[a-\tau, b]\right\}$ where $a$ and $b$ are constants, and $t_{n}$ is defined in (34). Following the same approach used in (51) we can write

$$
\begin{aligned}
\mathbb{P}\left\{\exists t_{n} \in[a-\tau, b]\right\}=1 & -\mathbb{P}\left\{t_{1}>b\right\} \\
& -\sum_{n=1}^{\infty} \mathbb{P}\left\{t_{n+1}>b, t_{n}<a-\tau\right\} .
\end{aligned}
$$

Defining $\theta_{n}=t_{n}+\tau$ we obtain $\mathbb{P}\left\{t_{n+1}>b, t_{n}<a-\tau\right\}=$ $\mathbb{P}\left\{\theta_{n}+\Delta_{n+1}>b, \theta_{n}<a\right\}$ and thus, given that $\theta_{n}$ and $\Delta_{n+1}$ are independent r.v.s, we can write

$$
\begin{aligned}
\mathbb{P} & \left\{t_{n+1}>b, t_{n}<a-\tau\right\} \\
& =\mathbb{P}\left\{\Delta_{n+1}>b-\theta_{n}, \theta_{n}<a\right\} \\
& =\int_{\xi<a} \mathbb{P}\left\{\Delta_{n+1}>b-x\right\} f_{\theta_{n}}(x) d x \\
& =\int_{\xi<a} e^{-\lambda(b-x)} f_{\theta_{n}}(x) d x .
\end{aligned}
$$

The p.d.f. of $\theta_{n}$ can be obtained from the complementary c.d.f. expression given in [35] as

$$
\begin{array}{r}
f_{\theta_{n}}(x)=\sum_{i=1}^{n} \widetilde{c}_{1 i} \frac{x^{n-i} e^{-\lambda x}}{\Gamma(n-i+1)} u(x) \\
+\sum_{j=1}^{n} \widetilde{c}_{2 j} \frac{x^{n-j} e^{-\mu x}}{\Gamma(n-j+1)} u(x) \\
\widetilde{c}_{1 i}=(-1)^{i-1}\left(\begin{array}{c}
i+n-2 \\
i-1
\end{array}\right) \frac{\lambda^{n} \mu^{n}}{(\mu-\lambda)^{n+i-1}} \\
\widetilde{c}_{2 j}=(-1)^{j-1}\left(\begin{array}{c}
j+n-2 \\
j-1
\end{array}\right) \frac{\lambda^{n} \mu^{n}}{(\lambda-\mu)^{n+j-1}}
\end{array}
$$

and therefore we derive

$$
\begin{aligned}
\mathbb{P}\left\{t_{n+1}>b, t_{n}<a-\tau\right\}= & e^{-\lambda b} \sum_{i=1}^{n} \widetilde{c}_{1 i} \frac{a^{n-i+1}}{(n-i+1) !} \\
& +\widetilde{c}_{2 i} \frac{\bar{\gamma}(n-i+1,(\mu-\lambda) a)}{(\mu-\lambda)^{n-i+1}} .
\end{aligned}
$$

The probability $\mathbb{P}\left\{\exists t_{n} \in[a-\tau, b]\right\}$ can be thus computed using (55) using (53) and (60).

\section{APPENDIX B}

In this section we provide the solutions of two integral forms useful for computation of the probabilities in Section V. We consider in particular integrals in the form $\int_{0}^{\infty} \bar{\gamma}(n, a+$ $b \tau) f_{\tau}(\tau) d \tau$ with $n \in \mathbb{N}, a, b, \mu \in \mathbb{R}, a, b, \mu>0$ and $b>\mu$. Considering the case in which $f_{\tau}(\tau)=\mu \exp (-\mu \tau)$, for $\tau \geq 0$ and zero otherwise, solving by parts we obtain

$$
\begin{aligned}
\int_{0}^{\infty} & \bar{\gamma}(n, a-b \tau) \mu \exp (-\mu \tau) d \tau \\
= & \frac{\mu}{b} \exp \left(-\frac{\mu}{b} a\right) \int_{0}^{a} \bar{\gamma}(n, x) \exp \left(\frac{\mu}{b} x\right) d x \\
= & \bar{\gamma}(n, a)-\gamma\left(n, a\left(1-\frac{\mu}{b}\right)\right)\left(\frac{b}{b-\mu}\right)^{n} \exp \left(-\frac{\mu}{b} a\right)
\end{aligned}
$$

Considering the case in which $f_{\tau}(\tau)=b \exp (-b \tau)$, for $\tau \geq 0$ and zero otherwise, we get the form

$$
\begin{aligned}
\int_{0}^{\infty} & \bar{\gamma}(n, a-b \tau) b \exp (-b \tau) d \tau \\
\quad= & \exp (-a) \int_{0}^{a} \bar{\gamma}(n, x) \exp (x) d x \\
\quad= & \bar{\gamma}(n, a)-\frac{a^{n}}{n !} \exp (-a)
\end{aligned}
$$




\section{ACKNOWLEDGMENT}

The authors wish to thank Prof. Marco Chiani for his valuable comments and suggestions, the Associate Editor and the anonymous reviewers for their constructive comments.

\section{REFERENCES}

[1] S. Kandeepan and A. Giorgetti, Cognitive Radio Techniques: Spectrum Sensing, Interference Mitigation and Localization, Mobile Communications Series, Artech House, New York/London, Sep. 2012.

[2] M. Mueck, et. al, "ETSI reconfigurable radio systems: status and future directions on software defined radio and cognitive radio standards", IEEE Commun. Mag., vol. 48, no. 9, pp. 78-86, Sep. 2010.

[3] A. Mariani, Spectrum sensing algorithms for cognitive radio applications, Ph.D. thesis, Philosophy Doctoral Program in Electronics, Computer Science and Telecommunications, University of Bologna, Cesena, Italy, 2013.

[4] H. Urkowitz, "Energy Detection of Unknown Deterministic Signals," Proc. of the IEEE, vol. 55, no. 4, pp. 523-531, Apr. 1967.

[5] A. Mariani, A. Giorgetti, and M. Chiani, "Effects of noise power estimation on energy detection for cognitive radio applications," IEEE Trans. Commun., vol. 59, no. 12, pp. 3410-3420, Dec. 2011.

[6] P. Wang, J. Fang, N. Han, and H. Li, "Multiantenna-assisted spectrum sensing for cognitive radio," IEEE Trans. Veh. Technol., vol. 59, no. 4, pp. 1791-1800, May 2010.

[7] A. Mariani, A. Giorgetti, and M. Chiani, "Test of independence for cooperative spectrum sensing with uncalibrated receivers," in Proc. IEEE Global Commun. Conf. (GLOBECOM 2012), pp. 1374-1379, Anaheim, CA, USA, Dec. 2012.

[8] T. Yucek and H. Arslan, "A survey of spectrum sensing algorithms for cognitive radio applications," IEEE Commun. Surveys \& Tuts., vol. 11, no. 1, pp. 116-130, Mar. 2009.

[9] T. C. Aysal, S. Kandeepan, R. Piesiewicz, "Cooperative Spectrum Sensing with Noisy Hard Decision Transmissions," in Proc. of the IEEE Int. Conf. on Commun. (ICC 2009), pp. 1-5, Dresden, Germany, Jun. 2009.

[10] S. Kandeepan, G. Baldini, and R. Piesiewicz, "Experimentally detecting IEEE $802.11 \mathrm{n} \mathrm{Wi-Fi} \mathrm{based} \mathrm{on} \mathrm{cyclostationarity} \mathrm{features} \mathrm{for} \mathrm{ultra-wide}$ band cognitive radios," in Proc. IEEE Int. Personal, Indoor and Mobile Radio Commun. Conf. (PIMRC 2009), pp. 2315-2319, Tokyo, Japan, Sep. 2009.

[11] S. Kandeepan, A. Giorgetti, M. Chiani, "Distributed 'Ring-Around' Sequential Spectrum Sensing for Cognitive Radio Networks," in Proc. IEEE Conf. on Commun. (ICC 2011), pp. 1-6, Kyoto, Japan, Jun. 2011.

[12] S. Kandeepan, A. B. Rahim, T. C. Aysal, and R. Piesiewicz, "Time Divisional and Time-Frequency Divisional Cooperative Spectrum Sensing," in Proc. Cognitive Radio Comm. Conf. (CROWNCOM 2009), pp. 1-6, Hannover, Germany, June 2009.

[13] Q. Zhao, S. Geirhofer, L. Tong, and B. M. Sadler, "Opportunistic spectrum access via periodic channel sensing," IEEE Trans. Signal Process., vol. 56, no. 2, pp. 785-796, Feb. 2008.

[14] S. Geirhofer, L. Tong, and B. M. Sadler, "Dynamic spectrum access in WLAN channels: Empirical model and its stochastic analysis," in Proc. First Int. Work. on Technology and Policy for Accessing Spectrum (TAPAS 2006), Boston, MA, USA, 2006.

[15] K. W. Choi and E. Hossain, "Opportunistic access to spectrum holes between packet bursts: a learning-based approach," IEEE Trans. on Wireless Commun., vol. 10, no. 8, pp. 2497-2509, Aug. 2011.

[16] A. D. Spaulding and G. H. Hagn, "On the Definition and Estimation of Spectrum Occupancy," IEEE Trans. Electromagnetic Compatibility, vol. 19, no. 3, pp. 269-280, Aug. 1977.

[17] S. Kandeepan, et al. "Spectrum Sensing for Cognitive Radios with Transmission Statistics: Considering Linear Frequency Sweeping," EURASIP J. Wirel Commun. Netw., vol. 6, Apr. 2010.

[18] H. Kim, and K. G. Shin, "In-band Spectrum Sensing in Cognitive Radio Networks: Energy Detection or Feature Detection," in Proc. Int. Conf. on Mobile Computing and Networking (MobiCom 2008), pp. 14-25, San Francisco, CA, USA, Sep. 2008.

[19] C. Cordeiro, K. Challapali, and M. Ghosh, "Cognitive PHY and MAC layers for dynamic spectrum access and sharing of TV bands," in Proc. ACM Int. Workshop on Technology and Policy for Accessing Spectrum (TAPAS 2006), Boston, MA, USA, Aug. 2006.

[20] C. Ghosh, S. Roy and B. Rao, "Modeling and Validation of Channel Idleness and Spectrum Availability for Cognitive Networks," IEEE J. Sel. Areas Commun., vol. 30, no. 10, pp. 2029-2039, Nov. 2012.
[21] S. Geirhofer, L. Tong, and B. M. Sadler, "Dynamic Spectrum Access in the Time Domain: Modeling and Exploiting White Space," IEEE Commun. Mag., vol. 45, no. 5, pp. 66-72, May 2007.

[22] J. Ma, X.-W. Zhou, and G. Y. Li, "A probability-based periodic spectrum sensing during secondary communication," IEEE Trans. Commun., vol. 58, no. 2, pp. 1291-1301, Apr. 2010.

[23] C. Ghosh, S. Pagadarai, D. P. Agrawal, and A. M. Wyglinski, "A framework for statistical wireless spectrum occupancy modeling," IEEE Trans. Wireless Commun., nol. 9, no. 1, pp. 38-44, Jan. 2010.

[24] M. Wellens and P. Mahonen, "Lessons Learned from an Extensive Spectrum Occupancy Measurement Campaign and a Stochastic Duty Cycle Model," Springer Mobile Networks and Applications Journal, vol. 15 , no. 3 , pp. $461-474,2010$.

[25] M. Lopez-Benitez, A. Umbert and F. Casadevall, "Evaluation of Spectrum Occupancy in Spain for Cognitive Radio Applications," in Proc. IEEE Veh. Technol. Conf. (VTC Spring 2009), pp. 1-5, Barcelona, Spain, Apr. 2009.

[26] H. Kim and K. G. Shin, "Efficient discovery of spectrum opportunities with MAC-layer sensing in cognitive radio networks" IEEE Trans. Mobile Comput., vol.7. no. 5, pp. 533-545, May 2008.

[27] M. Wellens, A. de Baynast, and P. Mahonen, "On the Performance of Dynamic Spectrum Access based on Spectrum Occupancy Statistics," IET Communications, vol. 2, no. 6, pp. 772-782, July 2008.

[28] S. Kandeepan, A. Sierra, J. Campos, and I. Chlamtac, "Periodic sensing in cognitive radios for detecting UMTS/HSDPA based on experimental spectral occupancy statistics," in Proc. IEEE Wireless Commun. and Netw. Conf. (WCNC 2010), pp. 1-6, Sydney, Australia, Apr. 2010.

[29] H. L. Van Trees, Detection, estimation, and modulation theory, New York: John Wiley \& Sons, 2004.

[30] ITU Handbook Teletraffic Engineering, ITU-D, Study Group 2, Jun. 2006, www.itu.int.

[31] T. Nguyen, B. L. Mark, and Y. Ephraim, "Spectrum Sensing Using a Hidden Bivariate Markov Model," IEEE Trans. on Wireless Commun., vol. 12, no. 9, pp. 4582-4591, Sep. 2013.

[32] L. Tang, Y. Chen, E. L. Hines, and M. S. Alouini, "Performance analysis of spectrum sensing with multiple status changes in primary user traffic," IEEE Commun. Letters, vol. 16, no. 6, pp. 874-877, Jun. 2012.

[33] T. Wang, Y. Chen, E. L. Hines, and B. Zhao, "Analysis of effect of primary user traffic on spectrum sensing performance," in Proc. Int. Conf. on Commun. and Networking in China, (ChinaCOM 2009), pp. 1-5, Beijing, China, Aug. 2009.

[34] B. Rassouli, A. Olfat, "Periodic spectrum sensing parameters optimization in cognitive radio networks", IET Communications, vol. 6 , no. 18, pp. 3329-3338, Dec. 2012.

[35] S. V. Amari and R. B. Misra, "Closed-form expressions for distribution of sum of exponential random variables," IEEE Trans. on Reliability, vol. 46 no. 4, pp. 519-522, Dec. 1997.

[36] F. Digham, M.-S. Alouini, and M. K. Simon, "On the Energy Detection of Unknown Signals over Fading Channels," IEEE Trans. Commun., vol. 55, no. 1, pp. 21-24, Jan. 2007.

[37] R. Deng, S. He, J. Chen, J. Jia, W. Zhuang, and Y. Sun, "Energyefficient spectrum sensing by optimal periodic scheduling in cognitive radio networks," IET Communications, vol. 6, no. 6, pp. 676-684, Jun. 2012.

[38] Y. Pei, et al., "Sensing-throughput tradeoff in cognitive radio networks: how frequently should spectrum sensing be carried out?" in Proc. IEEE Int. Symp. on Personal, Indoor and Mobile Radio Commun. (PIMRC 2007), pp. 1-5, Sep. 2007. 


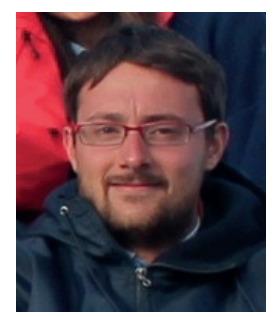

Andrea Mariani (S'10-M'14) received the B.Sc. degree in Biomedical Engineering (with honors) in 2006, the M.Sc. degree in Electronics and Telecommunications Engineering (with honors) in 2009, and the Ph.D. degree in Electronics, Computer Science and Telecommunications in 2013 from the University of Bologna, Italy. Currently, he is with the Center for Industrial Research on ICT (CIRI-ICT) of the University of Bologna. He has participated in several international projects, such as FP7-ICT EUWB, FP7-ICT SELECT and EDA-B CORASMA projects. His research interests include statistical signal processing and digital communications, with particular emphasis on spectrum sensing for cognitive radio networks and software defined radio. Dr. Mariani was a member of the Technical Program Committee of the Cognitive Radio and Networks Symposium at the IEEE Int. Conf. on Commun. (ICC 2013, ICC 2014 ICC 2015), the Cognitive Radio and Networks Symposium at the IEEE Global Commun. Conf. (Globecom 2013), and was a member of the local organization committee for the IEEE Int. Conf. on Ultra-WideBand (ICUWB 2011). He serves as reviewer for several IEEE Journals and Conferences, and has been named an Exemplary Reviewer 2013 for the IEEE WIRELESS COMMUNICATIONS LETTERS and for the IEEE COMMUNICATIONS LETTERS.

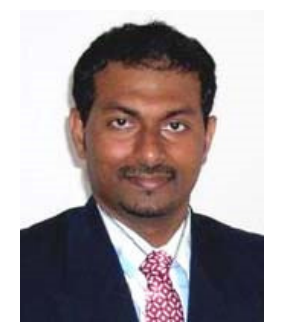

Sithamparanathan Kandeepan (SMIEEE) received his $\mathrm{PhD}$ in Electrical Engineering at the University of Technology, Sydney and is currently with the School of Electrical and Computer Engineering at the RMIT University, Melbourne. Kandeepan had previously worked with the Cooperative Research Center for Satellite Systems (CRCSS) in Sydney (2000-2004), with the National ICT Australia (NICTA) in Canberra (2004-2008), and with the CREATE-NET research center in Italy (20082011). Kandeepan was honored as an Exemplary Reviewer by the IEEE Communications Society in 2011 and is a Senior Member of the IEEE since 2009. He also serves as the Vice-Chair for the IEEE Technical Committee on Cognitive Networks. Kandeepans research interests are on wireless/mobile communications and applied statistical signal processing. He currently works on cognitive radios, cooperative networks and energy efficient wireless communication systems and had co-authored a book on 'Cognitive Radio Techniques' (2012) published by Artech House.

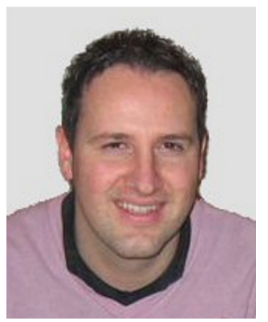

Andrea Giorgetti (S98-M04-SM13) received the Dr.Ing. degree (summa cum laude) in electronic engineering and the Ph.D. degree in electronic engineering and computer science, both from the University of Bologna, Italy, in 1999 and 2003, respectively. He was a Researcher with the National Research Council (2003-2005), and Assistant Professor at the University of Bologna (2006-2014). Since 2014 he is Associate Professor at the University of Bologna. During spring 2006, he was with the Laboratory for Information and Decision Systems (LIDS), Massachusetts Institute of Technology (MIT), Cambridge, MA, USA. Since then he is a Research Affiliate of LIDS, MIT. His research interests include ultra-wide bandwidth communications systems, active and passive localization, wireless sensor networks, and cognitive radio. Dr. Giorgetti was Technical Program Co-Chair of the Eleventh Int. Symp. on Wireless Commun. Systems (ISWCS 2014), Barcelona, Spain, Aug. 2014, the Cognitive Radio and Networks Symposium at the IEEE Int. Conf. on Commun. (ICC), Budapest, Hungary, June 2013, the Cognitive Radio and Networks Symposium at the IEEE Global Commun. Conf. (Globecom), Atlanta, GA, USA, December 2013, the Wireless Networking Symp. at the IEEE Int. Conf. on Commun. (ICC), Beijing, China, May 2008, and the MAC track at the IEEE Wireless Commun. and Networking Conf. (WCNC), Budapest, Hungary, April 2009. He is elected Secretary of the IEEE Communications Societys Radio Communications Technical Committee. He is an Editor for the IEEE TRANSACTIONS ON WIRELESS COMMUNICATIONS and for the IEEE COMMUNICATIONS LETTERS. 